\title{
Fast numerical methods for non-local operators
}

Hackbusch, Wolfgang ; Sauter, Stefan ; Schwab, Christoph

DOI: https://doi.org/10.4171/OWR/2004/33

Other titles: Abstracts from the workshop held July 4-10, 2004. Organized by Wolfgang Hackbusch, Stefan Sauter and Christoph Schwab.

Posted at the Zurich Open Repository and Archive, University of Zurich ZORA URL: https://doi.org/10.5167/uzh-21754

Journal Article

Originally published at:

Hackbusch, Wolfgang; Sauter, Stefan; Schwab, Christoph (2004). Fast numerical methods for non-local operators. Oberwolfach Reports, 1(3):1747-1788.

DOI: https://doi.org/10.4171/OWR/2004/33 


\title{
Mathematisches Forschungsinstitut Oberwolfach
}

Report No. 33/2004

\section{Fast Numerical Methods for Non-local Operators}

\author{
Organised by \\ Wolfgang Hackbusch (Leipzig) \\ Stefan Sauter (Zürich ) \\ Christoph Schwab (Zürich)
}

July 4th-July 10th, 2004

\section{Introduction by the Organisers}

The fast numerical treatment of non-local operators is an important challenge in many fields of mathematics and its applications. This includes classical Fredholm integral operators, retarded potentials for wave equations, inversion of (discretised) partial differential equations, solutions to the matrix Riccati equation, infinitesimal generators of step processes, non-local filter operators in image processing, or non-local potentials in quantum chemistry and process simulation. The terminology "fast" is related to the "Fast Fourier Transform" which allows to apply the (non-local) discrete Fourier transform of $N$ data points in $O(N \log N)$ operations instead of $O\left(N^{2}\right)$ operations.

With growing demand for reliable discretisation methods for such applications the need of fast numerical methods for non-local operators has increased rapidly worldwide since the mid 80th and we list below some of these methods:

(1) Cluster methods for the sparse representation of classical Fredholm integral operators.

(2) Multipole methods for the fast evaluation of Coulomb-type potentials.

(3) $\mathcal{H}$-matrices for the sparse representation of inverses of finite element discretisations of partial differential equations.

(4) Wavelet- and multiscale representations of non-local elliptic operators.

These methods allow to reduce the storage amount and the computational cost when applying a non-local operator to $O(N)$ data points from $O\left(N^{2}\right)$ to $O\left(N \log ^{\alpha} N\right)$, for some moderate $\alpha \sim 1$. This log-linear complexity is based on an approximate representation of non-local operators which are already afflicted with 
a consistency error stemming from an underlying numerical discretisation. The size of this additional error is controlled by suitable control parameters in such algorithms and the numerical analysis allows to choose them in an optimal way.

The efficiency of these newly developed numerical methods has generated a vivid research activity in this field of numerical analysis and new challenging problems are arising: For instance, we mention the ab-initio numerical solution of Schrödinger's equation for $N$ particle systems with far field interaction, where the numerical solution for very large $N$ is feasible only by using new fast algorithms for non-local operators. Among further novel applications is the optimal stopping of step-diffusion processes for which the infinitesimal generator of the process is non-local. The full potential of fast methods for such kinds of applications is not yet utilized and research activities are directed in these directions.

A further field of active research is the numerical solution of problems arising in electromagnetics. Such problems are described in a wide range of parameters (wave numbers, dielectric constants, etc.) by Maxwell's equation. Integral operators are often used to reduce the problem on unbounded domains to the compact surface of the scatterer. These operators depend on parameters, e.g., the wave number in a critical way and the fast algorithm are currently developed for such type of problems.

The goal of the (half) conference was to focus on the basic methodological problems such as

- numerical analysis of fast compression methods.

- algorithmic aspects of fast compression methods.

- fast algorithms for integral equations in electromagnetics.

- fast compression algorithms for new types of applications.

This Oberwolfach conference brought together 22 scientists in the field of fast numerical methods for non-local operators. The workshop had a clear mathematical focus on the systematic development of fast methods for new types of applications, on their algorithmic aspects and the relevant numerical analysis. A total of 17 presentations were given. The fact that after each talk there arose very stimulating and intense discussions show that the conference truly had workshop character where all participants profited from the talks and the discussions after the talks and in the breaks.

The topics of the talks can be categorized in the following areas:

- Discretisation by wavelets and Fast Multipole Methods

Wavelets allow the sparse discretisation of non-local operators due to the vanishing moment properties. Progress in the application of wavelets to (time-harmonic) electromagnetic problems for high wave numbers and for high-dimensional problems has been presented by the talks of R. Schneider, J. Tausch and E. Tyrtyshnikov. In O. Steinbach's talk Tearing and Interconnecting Domain Decomposition Methods, the FETI/BETI method was considered which is an efficient preconditioned iterative solver for finite element/boundary element domain decomposition methods. It was 
shown that dense matrices arising in BETI could be avoided by using the Fast Multipole Method.

\section{- $\mathcal{H}$-matrix arithmetics}

$\mathcal{H}$-matrices allow the sparse representation of non-local inverses of finite element discretisations of PDEs and to perform arithmetic operations such as matrix-matrix multiplication, matrix exponentials, etc. in loglinear complexity. In this field, $\mathcal{H}$-matrix LU based preconditioning of BEM equations have been presented by M. Bebendorf. In his lecture, L. Grasedyck presented adaptive coarsening strategies for $\mathcal{H}$-matrices. A further development in this area was presented by B. Khoromsikij in his talk on data sparse representations for multidimensional non-local operators.

\section{- Scattering Problems}

The numerical solution of inverse scattering problems can be based on an iteration process based on integral equations. The presentation integral equations and numerical solution of inverse scattering problems by R. Kress provided a survey on the newest developments in this area.

In his presentation, G. Monegato considered the problem of scattering at a T-junction. The arising integral operators are non-standard and results on their mapping properties and the numerical solution have been presented. In the talk of S. Sauter, the Galerkin method for Helmholtz' equation was analysed and error estimates which are explicit in the wave numbers have been presented. In his presentation, I. Graham presented a method which allows to use asymptotic information to compute diffraction coefficients in high frequency scattering.

\section{- Novel Applications}

In some talks, new applications in the field of non-local operators have been considered. S. Rjasanow has presented numerical solution methods for the non-local electrostatic problems arising in the modelling of large

biomolecules. C. Schwab gave a presentation on numerical solution of operator equations based on stochastic data. The computation of the $k$-th moments of the random solution can be based on high-dimensional integral equations and numerical methods for its solution have been presented.

\section{- hp Boundary Elements}

The discretisation of integral equations by $h p$ boundary element method enjoys exponential convergence rates. In the talk of E. Stephan on Schwarz methods for integral equations on surfaces, fast solvers for the arising systems of linear equations have been presented.

\section{- Convergence Theory for Boundary Elements Methods}

Besides the development of fast numerical methods for non-local operators there have been two talks on the stability and convergence theory for integral equations. W. Wendland gave a presentation on J. Radon's convergence proof of J. Neumann's method with double layer potentials 
while R. Hiptmair has presented a method for a stable coupling of integral equations in scattering. 


\section{Workshop: Fast Numerical Methods for Non-local Operators}

\section{Table of Contents}

Mario Bebendorf

H-Matrix LU-decomposition based preconditioners for BEM ........... 1749

Ivan G. Graham

Some hybrid asymptotic-numerical methods in high-frequency scattering . .1751

Lars Grasedyck

Adaptive Coarsening of Hierarchical Matrices ...................... 1752

Ralf Hiptmair

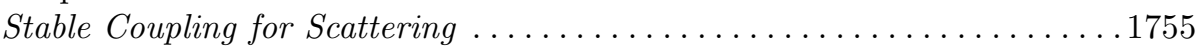

Boris N. Khoromskij (joint with Wolfgang Hackbusch)

Data-Sparse Representation of Multi-Diminsional Nonlocal Operators . . . 1758

Rainer Kress

Integral equations and numerical methods for inverse scattering problems .1759

Giovanni Monegato (joint with Renato Orta)

A hypersingular integral equation arising in a waveguide scattering problem 1762

Sergej Rjasanow

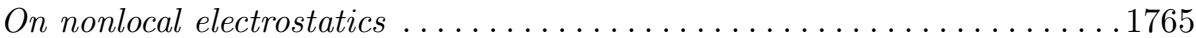

Stefan A. Sauter (joint with Annalisa Buffa)

Stabilisation of the acoustic single layer potential on non-smooth domains 1768

Reinhold Schneider (joint with Wolfgang Dahmen, Helmut Harbrecht)

Adaptive Wavelet Based Fast Solution of BEM ...................1771

Christoph Schwab (joint with Tobias von Petersdorff)

Numerical Solution of Operator Equations with Stochastic Data ........1771

Olaf Steinbach (joint with Ulrich Langer, Günther Of and Walter Zulehner)

Tearing and Interconnecting Domain Decomposition Methods ............ 1772

Ernst P. Stephan (joint with Florian Leydecker and Matthias Maischak)

Some Schwarz Methods for Integral Equations on Surfaces -

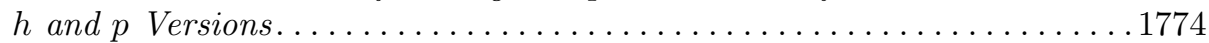

Johannes Tausch

A spectral method for integral formulations of potential and high-frequency

scattering problems ................................... 1775

Eugene Tyrtyshnikov (joint with Ivan Oseledets)

Matrix approximations and solvers using tensor products and non-standard wavelet transforms related to irregular grids . .................. 1778 
Wolfgang L. Wendland

On J. Radon's convergence proof of Neumann's method with double layer potentials ....................................... 1779 


\section{Abstracts \\ $H$-Matrix $L U$-decomposition based preconditioners for BEM MARIO BEBENDORF}

Stiffness matrices arising from integral formulations of elliptic problems are usually dense. However, the kernel function $\kappa$ of the arising integral operators can locally be approximated by degenerate functions, i.e.,

$$
\kappa(x, y) \approx \sum_{i=1}^{k} u_{i}(x) v_{i}(y),
$$

where $k$ is a small number. This idea originating from the fast multipole method [4] and panel clustering [7] today is looked at more from an algebraic point of view, since on the discrete level (1) means that appropriate blocks of the matrix $K$ can be approximated by matrices of low rank. This gave rise to the mosaic-skeleton method [10] and hierarchical matrices ( $\mathcal{H}$-matrices) $[5,6,3]$. By the latter it is not only possible to (approximatively) store dense matrices and multiply them by a vector with almost linear complexity, they also provide the usual (approximative) operations like matrix addition, multiplication and inversion with almost the same complexity. The basis for the efficiency of this class of matrices is a hierarchical partition of the matrix into blocks and the low-rank representation of each block. Instead of generating these low-rank approximants from degenerate kernel approximations, e.g, by the multipole expansion or interpolation, it is more efficient and convenient to use the adaptive cross approximation (ACA) algorithm [1,2], which finds such low-rank approximants from few of the original matrix entries.

When solving linear systems with these matrices iteratively the number of iterations needed to obtain a prescribed accuracy is mainly determined by the condition number of the coefficient matrix. A bad condition number may be caused by the mapping properties of the operator or by the underlying geometry or discretization. There are many different approaches to obtain a preconditioner $C$. In a recently published idea [8] an algebraic multigrid procedure is constructed for boundary element matrices. Another possibility is based on the mapping properties of the operator only, see [9]. Let $\mathcal{A}: V \rightarrow V^{\prime}$ be a $V$-coercive and $\mathcal{B}: V^{\prime} \rightarrow V$ a $V^{\prime}$ coercive operator. Then $\mathcal{A}$ and $\mathcal{B}^{-1}: V \rightarrow V^{\prime}$ both are $V$-coercive, i.e., there are constants $\alpha_{i}, \beta_{i}>0, i=1,2$, such that for all $v \in V$

$$
\alpha_{1}\|v\|_{V}^{2} \leq(\mathcal{A} v, v)_{L^{2}} \leq \alpha_{2}\|v\|_{V}^{2} \quad \text { and } \quad \beta_{1}\|v\|_{V}^{2} \leq\left(\mathcal{B}^{-1} v, v\right)_{L^{2}} \leq \beta_{2}\|v\|_{V}^{2} .
$$

From this it already follows that $\mathcal{A}$ and $\mathcal{B}^{-1}$ are spectrally equivalent, i.e,

$$
\frac{\alpha_{1}}{\beta_{2}}\left(\mathcal{B}^{-1} v, v\right) \leq(\mathcal{A} v, v) \leq \frac{\alpha_{2}}{\beta_{1}}\left(\mathcal{B}^{-1} v, v\right) \quad \text { for all } v \in V .
$$

Hence, for preconditioning the single layer potential operator of the Laplacian for instance the hypersingular operator can be used. Since both operators can be approximated using ACA, one could easily obtain an efficient preconditioner. 
Although the idea of using operators with inverse mapping properties is attractive, the preconditioning effect can only be observed asymptotically, i.e., for fixed problem sizes $n$ the condition number might still be large.

The approach we are going to pursue is to use an approximative $L U$ decomposition

$$
C:=L U
$$

of the coefficient matrix $A \approx L U$, where the two triangular factors $L$ and $U$ are stored in $\mathcal{H}$-matrix format. Depending on the prescribed accuracy of the $L U$ decomposition the condition number can be controlled also for fixed problem sizes. A low precision $L U$ decomposition will be sufficient to guarantee spectral equivalence. The arising equations with coefficient matrix $C$ can then be solved by forward/backward substitution with the same complexity as the $\mathcal{H}$-matrix-vector multiplication. The setup of the factors $L$ and $U$ can be done with complexity $n \log ^{6} n$. Since constants are much smaller compared with the complexity of build-

ing $A$, the time for decomposing $A$ can be neglected. This is especially true, if the $L U$ decomposition is computed with low precision.

\section{REFERENCES}

[1] M. Bebendorf: Approximation of boundary element matrices. Numer. Math. 86, 565-589, 2000.

[2] M. Bebendorf and S. Rjasanow: Adaptive Low-Rank Approximation of Collocation Matrices. Computing 70, 1-24, 2003.

[3] L. Grasedyck and W. Hackbusch: Construction and arithmetics of $\mathcal{H}$-matrices, Computing 70: 295-334, 2003.

[4] L. Greengard and V. Rokhlin: A new version of the fast multipole method for the Laplace equation in three dimensions. Acta numerica, 1997, pages 229-269. Cambridge Univ. Press, Cambridge, 1997.

[5] W. Hackbusch: A sparse matrix arithmetic based on $\mathcal{H}$-matrices. I. Introduction to $\mathcal{H}$ matrices. Computing 62, 89-108, 1999.

[6] W. Hackbusch and B. N. Khoromskij: A sparse $\mathcal{H}$-matrix arithmetic. II. Application to multi-dimensional problems. Computing 64, 21-47, 2000.

[7] W. Hackbusch and Z. P. Nowak: On the fast matrix multiplication in the boundary element method by panel clustering. Numer. Math. 54, 463-491, 1989.

[8] U. Langer, D. Pusch and S. Reitzinger: Efficient preconditioners for boundary element matrices based on grey-box algebraic multigrid methods, Int. J. Numer. Meth. Engng. 58, 1937-1953, 2003.

[9] O. Steinbach and W. Wendland: The construction of some efficient preconditioners in the boundary element method, Adv. Comput. Math. 9, 191-216, 1998.

[10] E. Tyrtyshnikov: Mosaic-skeleton approximations. Calcolo 33, 47-57 (1998), 1996. 


\section{Some hybrid asymptotic-numerical methods in high-frequency scattering \\ IVAN G. GRAHAM}

In this talk we presented two techniques for combining asymptotic information with numerical methods in order to produce wave-number robust solution methods for some high-frequency problems in acoustic and electromagnetic scattering.

The first part of the talk (joint work with B.D. Bonner and V.P. Smyshlyaev [3]) concerned the computation of diffraction coefficients for the scattering of highfrequency waves by conical scatterers. The diffraction coefficient gives the coefficient of the principal term in the asymptotic expansion of the diffracted component of the scattered wave as the wave number $k \rightarrow \infty$. The computation of the diffraction coefficient in the case of a conical scatterer can be reduced to the solution of a family of homogeneous boundary value problems for the LaplaceBeltrami-Helmholtz equation on a portion of the unit sphere bounded by a simple closed contour (in fact the intersection of the sphere with the conical scatterer). The diffraction coefficient may be determined by then integrating the resulting solutions with respect to the wave number ([1]).

In this part of the talk we discussed the numerical computation of the diffraction coefficients using the boundary integral method, with the classical double layer potential approach. We gave an analysis of the scalar integral equation arising in acoustic scattering, which shows its relation to the corresponding integral equation for the planar Helmholtz equation. This allows us to prove, using the results of [2], optimal convergence for piecewise polynomial collocation methods of arbitrary order even when the scatterer has non-smooth cross-section. We also derived efficient quadrature techniques for assembling the boundary element matrices. In practice we employ an $h-p$ approximation scheme, which converges with exponential order.

The scattering of electromagnetic waves was also discussed; the resulting system of integral equations can be analysed by similar techniques to those used for the acoustic case. We illustrated this part with computations on both smooth and non-smooth scatterers for both the acoustic and electromagnetic cases.

In the second part of the talk we described joint work in progress, with $\mathrm{V}$. Dominguez, on methods for incorporating asymptotic information into the design of ansatz functions so that standard numerical methods (such as the Galerkin method) work well for both low and high frequency applications. We illustrated this for the case of acoustic scattering from a circular object, where diffraction due to grazing incidence at the tangency points as well as simple reflections are the main features which have to be taken account of.

\section{REFERENCES}

[1] V.M.Babich, V.P.Smyshlyaev, D.Dement'ev and B.A.Samokish, SIAM J. Appl. Math. 60(2000), 536-573. 
[2] J.Elschner and I.G. Graham, Numerical methods for integral equations of Mellin type, in Numerical Analysis 2000, Volume 6: Ordinary differential and Integral Equations, C.T.H. Baker, J.D. Pryce, G.Vanden Berghe and G.Monegato, eds. Elsevier, Amsterdam, 2001, also J. Comp. Appl. Math. 125 (2000), 423-437.

[3] B.D. Bonner. I.G.Graham and V.P.Smyshlyaev, The computation of conical diffraction coefficients in high-frequency acoustic wave scattering, to appear in SIAM J. Numer. Anal. Preprint: http://www.maths.bath.ac.uk/ ${ }^{\sim}$ igg

\section{Adaptive Coarsening of Hierarchical Matrices LARS GRASEDYCK}

The efficient treatment of dense matrices arising, e.g., from the finite element discretisation of integral operators requires special compression techniques. In this talk we use a hierarchical low-rank approximation, the so-called $\mathcal{H}$-matrix, that approximates the dense stiffness matrix in admissible blocks (corresponding to subdomains where the underlying kernel function is smooth) by low rank matrices. The low rank matrices are assembled either by kernel interpolation [4], adaptive cross approximation $[14,2]$ or multipole expansions [9].

In the talk we develop an algorithm that can determine a coarser block structure that minimises the storage requirements (enhanced compression) and speeds up the (formatted) arithmetic of $\mathcal{H}$-matrices. This coarse approximation is done adaptively and on-the-fly to a given accuracy such that the matrix is assembled with minimal storage requirements while keeping the desired approximation quality. It can be regarded as an algebraic extension of the weak admissibility condition [12]. Moreover, the approximate inverse of a rather coarse approximation to the stiffness matrix turns out to be a good preconditioner.

The $\mathcal{H}$-matrix format is presented in a series of papers $[10,11,6,8]$ that describe the construction as well as the arithmetic in this format. Our coarsening strategy does not improve the asymptotic bounds for the complexity of the arithmetic, but it minimises the constants.

The (hierarchical) partition of the matrix into blocks that allow for a low-rank approximation is not unique. Even the question whether or not a single block is suitable for a low-rank approximation is non-trivial. Typically, one demands that the singular values of the matrix block decay exponentially and ensures this by sufficient conditions (standard admissibility, weak admissibility).

For a rather coarse approximation of a matrix block an exponential decay of the singular values is not necessary. Therefore, standard (or weak) admissibility conditions for the blocks are too restrictive. The partitions generated by these sufficient conditions serve as input for our algorithm that coarsens the structure.

A first recompression method is commonly used when applying sub-optimal rank revealing algorithms: each assembled block in the low-rank format is immediately decomposed by the SVD. Since the block is already given in factorised form, the SVD of such an $n \times m$ rank $k$ matrix can be computed in $\mathcal{O}\left(k^{2}(n+m)\right)$ [8]. All singular values $\sigma_{i}$ with $\sigma_{i} \leq \varepsilon \sigma_{1}$ are discarded and the rank thereby 


\begin{tabular}{l|ccc|ccc}
$n$ & $\begin{array}{c}\text { Assembly } \\
{[\text { Sec. }]}\end{array}$ & $\begin{array}{c}\text { Store } \\
{[\mathrm{KB} / \mathrm{DoF}]}\end{array}$ & $\left\|I-A_{\mathcal{H}}^{-1} A\right\|$ & $\begin{array}{c}\text { Coarsen } \\
{[\text { Sec. }]}\end{array}$ & $\begin{array}{c}\text { Store } \\
{[\mathrm{KB} / \mathrm{DoF}]}\end{array}$ & $\left\|I-\tilde{A}_{\mathcal{H}}^{-1} A\right\|$ \\
\hline 2024 & 4 & 2.8 & $4.3 \cdot 10^{-3}$ & 2 & 1.4 & $1.8 \cdot 10^{-2}$ \\
8192 & 24 & 3.3 & $4.4 \cdot 10^{-3}$ & 10 & 2.1 & $1.0 \cdot 10^{-2}$ \\
32768 & 120 & 4.9 & $2.3 \cdot 10^{-3}$ & 49 & 3.1 & $6.0 \cdot 10^{-3}$ \\
131072 & 641 & 6.2 & $1.5 \cdot 10^{-3}$ & 241 & 4.4 & $2.4 \cdot 10^{-3}$
\end{tabular}

TABLE 1. Initial approximation (left) and coarsened $\mathcal{H}$-matrix (right).

reduced. The parameter $\varepsilon$ is the same that we use in the initial assembly of the block, e.g., an analytic error bound or a heuristic stopping criterion.

While the first recompression reduces the blockwise rank of the $\mathcal{H}$-matrix approximation, the following second recompression aims at a coarsening of the entire block structure of the $\mathcal{H}$-matrix. The reason why the initial block structure is not optimal is threefold:

First, the parameter $\eta$ from the admissibility condition might be too small (and it enters the complexity estimates in the power 6). For the discretisation this is not crucial but for the $\mathcal{H}$-matrix arithmetic it is. Our recompression scheme will automatically choose the right blocks, so that only the extra time for the assembly of the stiffness matrix is increased, but the storage requirements stay the same independently of $\eta$.

Second, blocks that are not admissible might be regarded as admissible, because the standard admissibility condition is sufficient but not necessary. This was first observed in [12] under the name weak admissibility.

Third, the block cluster tree based on the given cluster tree does not take the accuracy of the discretisation and compression into account. Asymptotically ( $k$ large enough or $\varepsilon$ small enough) the optimal block structure might be that from the standard admissibility, but for all practical purposes the optimal structure is coarser.

As a numerical example we consider the Galerkin discretisation by piecewise constant basisfunctions of the double layer potential of the Laplacian on the unit sphere. The initial approximation including the first recompression is assembled by automatic quadrature [5] in the nearfield and ACA [2] in the farfield. We denote this matrix by $A_{\mathcal{H}}$. A sufficiently accurate approximation to the full discrete matrix is stored in $A$. Table 1 contains the results for the initial approximation and first recompression. The second recompression $\tilde{A}_{\mathcal{H}}$ by adaptive coarsening of the initial $\mathcal{H}$-matrix approximation reduces the storage requirements from 6.2 down to $4.4 \mathrm{~KB}$ per degree of freedom, cf. Table 1.

At last, we consider the complexity of the formatted $\mathcal{H}$-matrix arithmetic for a more realistic geometry, namely the single layer potential operator of the Laplacian on a crank shaft discretised with 113152 piecewise constant basis functions. Here, the assembly takes 131.5 seconds, the accurate recompression 59 seconds and a rather coarse recompression 40 seconds. The rather coarse recompressed matrix 
can be decomposed by a Cholesky decomposition along the lines of $[13,7,1]$ in 24 seconds and allows us to solve the system by GMRES in 8 steps (11 seconds). The computations were performed on a SUN UltraSPARC with a $900 \mathrm{MHz} \mathrm{CPU}$ using the standard HLiB library [3] for hierarchical matrices.

\section{REFERENCES}

[1] M. Bebendorf. Hierarchical LU decomposition based preconditioners for BEM. Technical Report 28, Max Planck Institute for Mathematics in the Sciences, 2004.

[2] M. Bebendorf and S. Rjasanov. Adaptive Low-Rank Approximation of Collocation Matrices. Computing, 70:1-24, 2003.

[3] S. Börm and L. Grasedyck. HLiB - a library for $\mathcal{H}$ - and $\mathcal{H}^{2}$-matrices, 1999. Available at: http://www.hlib.org

[4] S. Börm and L. Grasedyck. Low-rank approximation of integral operators by interpolation. Computing, 72:325-332, 2004.

[5] S. Erichsen, S. Sauter. Efficient automatic quadrature in 3-d Galerkin BEM. Comput. Meth. Appl. Mech. Eng. 157: 215-224, 1998.

Computer Methods in Applied Mechanics and Engineering 157, Elsevier, 215-224 (1998).

[6] L. Grasedyck. Theorie und Anwendungen Hierarchischer Matrizen. PhD thesis, Universität Kiel, 2001.

[7] L. Grasedyck. Adaptive Recompression of $\mathcal{H}$-Matrices for BEM. Accepted for Computing.

[8] L. Grasedyck and W. Hackbusch. Construction and arithmetics of $\mathcal{H}$-matrices. Computing, 70:295-334, 2003.

[9] L. Greengard and V. Rokhlin. A new version of the fast multipole method for the Laplace in three dimensions. In Acta Numerica 1997, pages 229-269. Cambridge University Press, 1997.

[10] W. Hackbusch. A sparse matrix arithmetic based on $\mathcal{H}$-matrices. Part I: Introduction to $\mathcal{H}$-matrices. Computing, 62:89-108, 1999.

[11] W. Hackbusch and B. Khoromskij. A sparse matrix arithmetic based on $\mathcal{H}$-matrices. Part II: Application to multi-dimensional problems. Computing, 64:21-47, 2000.

[12] W. Hackbusch, B. Khoromskij, and R. Kriemann. Hierarchical matrices based on a weak admissibility criterion. Technical Report 2, Max Planck Institute for Mathematics in the Sciences, 2003. Submitted to Computing.

[13] M. Lintner. The eigenvalue problem for the 2d Laplacian in $\mathcal{H}$-matrix arithmetic and application to the heat and wave equation. Computing, 72:293-323, 2004.

[14] E. Tyrtyshnikov. Incomplete cross approximation in the mosaic-skeleton method. Computing, 64:367-380, 2000. 


\section{Stable Coupling for Scattering \\ RALF HiptMair}

\section{INTRODUCTION}

For a bounded scatterer $\Omega^{-} \subset \mathbb{R}^{3}$ with Lipschitz-continuous boundary $\Gamma:=$ $\partial \Omega^{-}$and complement $\Omega^{+}:=\bar{\Omega}^{-}$we consider the following transmission problem for the Helmholtz equation

$$
\begin{gathered}
\Delta u+k(\mathbf{x})^{2} u=f \quad \text { in } \Omega^{-} \quad, \quad \Delta u+\kappa^{2} u=0 \quad \text { in } \Omega^{+} \\
{\left[\gamma_{0} u\right]_{\Gamma}=0 \quad \text { across } \Gamma \quad, \quad\left[\gamma_{1} u\right]_{\Gamma}=0 \text { across } \Gamma,} \\
\frac{\partial}{\partial r} u(\mathbf{x})-i \kappa u(\mathbf{x})=o\left(r^{-1}\right) \quad \text { for } r:=|\mathbf{x}| \rightarrow \infty \text { uniformly } .
\end{gathered}
$$

Here $\gamma_{0}$ and $\gamma_{1}$ stand for the Dirichlet and Neumann traces, respectively, and $[\cdot]_{\Gamma}$ denotes the jump across $\Gamma$. The transmission problem (1) models the propagation of time-harmonic sound waves impinging on a penetrable object [6, Sect. 2.1]. It is known that (1) has a unique solution $u \in H_{\text {loc }}\left(\Delta, \mathbb{R}^{3}\right)$, cf. [6, Sect.3.2].

Definition 1. A distribution $u \in H_{\mathrm{loc}}^{1}\left(\Omega^{ \pm}\right)$is called exterior/interior Helmholtz solution, if $\Delta u+\kappa^{2}=0$ in $\Omega^{ \pm}$and (2) holds at $\infty$.

\section{BoundaRY INTEGRAL OPERATORS}

Based on single and double layer potentials $\Psi_{\mathrm{SL}}^{0}$ and $\Psi_{\mathrm{DL}}^{0}$ for the Helmholtz kernel $G(\mathbf{z}):=\exp (i \kappa|\mathbf{z}|)(4 \pi|\mathbf{z}|)^{-1}$ we can introduce the following four continuous boundary operators

$$
\begin{array}{llll}
\mathrm{V}_{\kappa}:=\{\Gamma\}_{\Gamma} & : H^{-\frac{1}{2}}(\Gamma) \mapsto H^{\frac{1}{2}}(\Gamma) \quad, \quad \mathrm{W}_{\kappa}:=\{\Gamma\}_{\Gamma} \quad: H^{\frac{1}{2}}(\Gamma) \mapsto H^{-\frac{1}{2}}(\Gamma), \\
\mathrm{K}_{\kappa}:=\{\Gamma\}_{\Gamma} \quad: H^{\frac{1}{2}}(\Gamma) \mapsto H^{\frac{1}{2}}(\Gamma) \quad, \quad \mathrm{K}_{\kappa}^{\prime}:=\{\Gamma\}_{\Gamma} \quad: H^{-\frac{1}{2}}(\Gamma) \mapsto H^{-\frac{1}{2}}(\Gamma) .
\end{array}
$$

For details please consult $[11$, Ch. 3$]$ and $[9$, Ch. $7 \& 9]$, also for a proof of the following theorem.

Theorem 2. The operators $\mathrm{V}_{\kappa}:: H^{-\frac{1}{2}}(\Gamma) \mapsto H^{\frac{1}{2}}(\Gamma)$ and $\mathrm{W}_{\kappa}: H^{\frac{1}{2}}(\Gamma) \mapsto H^{-\frac{1}{2}}(\Gamma)$ are coercive ${ }^{1}$.

Throughout, I assume that the duality pairing on $H^{-\frac{1}{2}}(\Gamma) \times H^{\frac{1}{2}}(\Gamma)$ is induced by the Hermitian sesqui-linear form $(\varphi, v)_{\Gamma}:=\int_{\Gamma} \varphi \bar{v} \mathrm{~d} S$.

A crucial tool for the coupling of a variational equations in $\Omega^{-}$and boundary integral equations on $\Gamma$ are the Calderón projectors [12]

$$
\mathrm{P}_{-}:=\left(\begin{array}{cc}
\frac{1}{2} I d-\mathrm{K}_{\kappa} & \mathrm{V}_{\kappa} \\
\mathrm{W}_{\kappa} & \frac{1}{2} I d+\mathrm{K}_{\kappa}^{\prime}
\end{array}\right) \quad, \quad \mathrm{P}_{+}:=\left(\begin{array}{cc}
\frac{1}{2} I d+\mathrm{K}_{\kappa} & -\mathrm{V}_{\kappa} \\
-\mathrm{W}_{\kappa} & \frac{1}{2} I d-\mathrm{K}_{\kappa}^{\prime}
\end{array}\right) .
$$

\section{Theorem 3.}

$$
\text { Range }\left(\mathrm{P}_{ \pm}\right)=H_{C D}^{ \pm}(\Gamma):=\left\{\left(\gamma_{0}^{ \pm} u, \gamma_{1}^{ \pm} u\right): \Delta u+\kappa^{2} u=0 \text { in } \Omega^{ \pm} \text {and }(2)\right\} .
$$

\footnotetext{
${ }^{1}$ Here, coercivity means that a Gårding inequality holds.
} 


\section{Classical symmetric COUpling}

A solution of (1) satisfies for all $v \in H^{1}\left(\Omega^{-}\right)$

$$
a(u, v):=\int_{\Omega^{-}} \operatorname{grad} u \cdot \operatorname{grad} \bar{v}-k(\mathbf{x})^{2} u \bar{v} \mathrm{~d} \mathbf{x}-\left(\gamma_{1}^{-} u, \gamma_{0}^{-} v\right)_{\Gamma}=\int_{\Omega^{-}} f \bar{v} \mathrm{~d} \mathbf{x} .
$$

The symmetric coupling approach due to Costabel [7] uses the exterior Calderón projector and the transmission conditions of (1) and obtains: seek $u \in H^{1}\left(\Omega^{-}\right)$, $\lambda \in H^{-\frac{1}{2}}(\Gamma)$ such that for all $v \in H^{1}\left(\Omega^{-}\right), \varphi \in H^{-\frac{1}{2}}(\Gamma)$

$$
\begin{aligned}
& a(u, v)+\left(\mathrm{W}_{\kappa} \gamma_{0}^{-} u, \gamma_{0}^{-} v\right)_{\Gamma}-\left(\left(\frac{1}{2} I d-\mathrm{K}_{\kappa}^{\prime}\right) \lambda, \gamma_{0}^{-} v\right)_{\Gamma}=f(v), \\
& \left(\varphi,\left(\frac{1}{2} I d-\mathrm{K}_{\kappa}\right) \gamma_{0}^{-} u\right)_{\Gamma}+\mathrm{V}_{\kappa}\left(\varphi, \mathrm{V}_{\kappa} \lambda\right)_{\Gamma} \quad=0 .
\end{aligned}
$$

The sesqui-linear form underlying (4) is coercive, but fails to be injective, if $\kappa^{2}$ coincides with a Dirichlet eigenvalue of $-\Delta$ in $\Omega^{-}$. This is the phenomenon of spurious resonances: the failure of a boundary integral equation formulation of (1) to possess a unique solution despite the unique solvability of (1).

A remedy is offered by the so-called combined field integral equations (CFIE) $[1,5]$. A simple version relies on a complex linear combination of the two exterior Calderón identities, but fails to produce a coercive variational problem in $L^{2}(\Gamma)$ in case $\Gamma$ has edges and corners.

\section{TRAnSFORMED TRACES}

More elaborate CFIE respect the natural trace spaces and use a regularizing compact linear operator $\mathrm{M}: H^{-\frac{1}{2}}(\Gamma) \mapsto H^{\frac{1}{2}}(\Gamma)$ that satisfies $(\varphi, \mathrm{M} \varphi)_{\Gamma}>0 \Leftrightarrow$ $\varphi \neq 0$.

For theoretical purposes regularizing operators have been introduced used by Panich [10], whereas their practical use in CFIE context is discussed in [2, 3, 4], see also the abstract by $\mathrm{S}$. Sauter. The simplest choice for $\mathrm{M}$ is $\mathrm{M}=\left(-\Delta_{\Gamma}+I d\right)^{-1}$ : $H^{-1}(\Gamma) \mapsto H^{1}(\Gamma)$, which means, for $\varphi \in H^{-\frac{1}{2}}(\Gamma)$,

$$
\left(\operatorname{grad}_{\Gamma} \mathrm{M} \varphi, \operatorname{grad}_{\Gamma} q\right)_{\Gamma}+(\mathrm{M} \varphi, q)_{\Gamma}=(\varphi, q)_{\Gamma} \quad \forall q \in H^{1}(\Gamma) .
$$

The operator $\mathrm{M}$ is utilized for the following bijective transformation of traces

$$
\mathrm{\top}:=\left(\begin{array}{cc}
I d & i \eta \mathrm{M} \\
i \eta & I d
\end{array}\right): H^{\frac{1}{2}}(\Gamma) \times H^{-\frac{1}{2}}(\Gamma) \mapsto H^{\frac{1}{2}}(\Gamma) \times H^{-\frac{1}{2}}(\Gamma),
$$

with regularization parameter $\eta>0$.

\section{Lemma 4.}

$$
\operatorname{Range}\left(\mathrm{TP}_{-}\right) \cap\left(\{0\} \times H^{-\frac{1}{2}}(\Gamma)\right)=\{0\}
$$




\section{Stabilized COUPLED PROBlem}

Applying $\mathrm{T}$ to the interior Calderón indentity, we arrive at a transformed exterior Calderón indentity for traces of exterior Helmholtz solutions.

$$
\left(\begin{array}{c}
\gamma_{0}^{+} u \\
\gamma_{1}^{+} u
\end{array}\right)=\left(\begin{array}{cc}
\frac{1}{2} I d+\mathrm{K}_{\kappa}-i \eta \mathrm{M} \circ \mathrm{W}_{\kappa} & -\mathrm{V}_{\kappa}-i \eta\left(\frac{1}{2} I d+\mathrm{K}_{\kappa}^{\prime}\right) \\
-\mathrm{W}_{\kappa}+i \eta\left(K-\frac{1}{2} I d\right) & \frac{1}{2} I d-\mathrm{K}_{\kappa}^{\prime}-i \eta \mathrm{V}_{\kappa}
\end{array}\right)\left(\begin{array}{c}
\gamma_{0}^{+} u \\
\gamma_{1}^{+} u
\end{array}\right)
$$

Combining (7) this with (3) yields the variational problem: seek $u \in H^{1}\left(\Omega^{-}\right)$, $\lambda \in H^{-\frac{1}{2}}(\Gamma)$ such that for all $v \in H^{1}\left(\Omega^{-}\right), \varphi \in H^{-\frac{1}{2}}(\Gamma)$

$$
\begin{gathered}
a(u, v)+\left(\mathrm{W}_{\kappa} \gamma_{0}^{-} u, \gamma_{0}^{-} v\right)_{\Gamma}-i \eta\left(\left(\mathrm{K}_{\kappa}-\frac{1}{2} I d\right) \gamma_{0}^{-} u, \gamma_{0}^{-} v\right)_{\Gamma} \\
-\left(\left(\frac{1}{2} I d-\mathrm{K}_{\kappa}^{\prime}\right) \lambda, \gamma_{0}^{-} v\right)_{\Gamma}+i \eta\left(\mathrm{V}_{\kappa} \lambda, \gamma_{0}^{-} v\right)_{\Gamma}
\end{gathered}
$$

Compared to (4), all extra terms in (8) are compact, which ensures coercivity. Moreover, from Lemma 4 we can conclude uniqueness of solutions:

Theorem 5. Solutions $(u, \lambda) \in H^{1}\left(\Omega^{-}\right) \times H^{-\frac{1}{2}}(\Gamma)$ of (8) are unique.

Let us assume the choice (5) for $\mathrm{M}$. In order to obtain a variational problem amenable to boundary element Galerkin discretization, we have to weed out products of non-local operators by introducing the new unknown

$$
p:=i \eta \mathrm{M}\left(\mathrm{W}_{\kappa} \gamma_{0}^{-} u+\left(\frac{1}{2} I d+\mathrm{K}_{\kappa}^{\prime}\right) \lambda\right) \in H^{1}(\Gamma) .
$$

Note that, actually, $p=0$ Then, (8) can be equivalently stated as: seek $u \in$ $H^{1}\left(\Omega^{-}\right), \lambda \in H^{-\frac{1}{2}}(\Gamma), p \in H^{1}(\Gamma)$ such that

$$
\begin{aligned}
& a(u, v)+\left(\mathrm{W}_{\kappa} \gamma_{0}^{-} u, \gamma_{0}^{-} v\right)_{\Gamma}-i \eta\left(\left(\mathrm{K}_{\kappa}-\frac{1}{2} I d\right) \gamma_{0}^{-} u, \gamma_{0}^{-} v\right)_{\Gamma} \\
&-\left(\left(\frac{1}{2} I d-\mathrm{K}_{\kappa}^{\prime}\right) \lambda, \gamma_{0}^{-} v\right)_{\Gamma}+i \eta\left(\mathrm{V}_{\kappa} \lambda, \gamma_{0}^{-} v\right)_{\Gamma}=f(v), \\
&\left(\varphi,\left(\frac{1}{2} I d-\mathrm{K}_{\kappa}\right) \gamma_{0}^{-} u\right)_{\Gamma}+\left(\varphi, i \eta \mathrm{MW}_{\kappa} \gamma_{0}^{-} u\right)_{\Gamma} \\
&+\left(\varphi, \mathrm{V}_{\kappa} \lambda\right)_{\Gamma}+(\varphi, p)_{\Gamma}=0, \\
&-i \eta\left(\mathrm{W}_{\kappa} \gamma_{0}^{-} u\right) q-\left(\left(\frac{1}{2} I d+\mathrm{K}_{\kappa}^{\prime}\right) \lambda, q\right)_{\Gamma} \\
&+\left(\operatorname{grad}_{\Gamma} p, \operatorname{grad}_{\Gamma} q\right)_{\Gamma}+(p, q)_{\Gamma}=0,
\end{aligned}
$$

for all $v \in H^{1}\left(\Omega^{-}\right), \varphi \in H^{-\frac{1}{2}}(\Gamma)$, and $q \in H^{1}(\Gamma)$. Now, by standard theory, a combined Galerkin finite element and boundary element discretization of (10) will yield asymptotically quasi-optimal solutions. 


\section{REFERENCES}

[1] H. Brakhage And P. Werner, Ueber das Dirichletsche Außenraumproblem für die Helmholtzsche Schwingungsgleichung, Arch. der Math., 16 (1965), pp. 325-329.

[2] A. Buffa And R. Hiptmair, Galerkin boundary element methods for electromagnetic scattering, in Topics in Computational Wave Propagation. Direct and inverse Problems, M. Ainsworth, P. Davis, D. Duncan, P. Martin, and B. Rynne, eds., vol. 31 of Lecture Notes in Computational Science and Engineering, Springer, Berlin, 2003, pp. 83-124.

[3] - A coercive combined field integral equation for electromagnetic scattering, SIAM J. Numer. Anal., 40 (2004), pp. 621-640.

[4] A. Buffa And S. SAuter, Stabilisation of the acoustic single layer potential on non-smooth domains, Preprint 19-2003, Institut für Mathematik, Universität Zürich, Zürich, Switzerland, 2003.

[5] A. Burton And G. Miller, The application of integral methods for the numerical solution of boundary value problems, Proc. R. Soc. London, Ser. A, 232 (1971), pp. 201-210.

[6] D. Colton And R. Kress, Inverse Acoustic and Electromagnetic Scattering Theory, vol. 93 of Applied Mathematical Sciences, Springer, Heidelberg, 2nd ed., 1998.

[7] M. Costabel, Symmetric methods for the coupling of finite elements and boundary elements, in Boundary Elements IX, C. Brebbia, W. Wendland, and G. Kuhn, eds., SpringerVerlag, Berlin, 1987, pp. 411-420.

[8] R. Hiptmair and P. Meury, Stable FEM-BEM coupling for acoustic scattering, tech. report, SAM, ETH Zürich, Zürich, Switzerland, 2004. In preparation.

[9] W. McLean, Strongly Elliptic Systems and Boundary Integral Equations, Cambridge University Press, Cambridge, UK, 2000.

[10] O. PANICH, On the question of the solvability of the exterior boundary-value problems for the wave equation and maxwell's equations, Usp. Mat. Nauk., 20A (1965), pp. 221-226. In Russian.

[11] S. Sauter and C. Schwab, Randelementmethoden, BG Teubner, Stuttgart, 2004.

[12] T. von Petersdorff, Boundary integral equations for mixed Dirichlet, Neumann and transmission problems, Math. Meth. Appl. Sci., 11 (1989), pp. 185-213.

\section{Data-Sparse Representation of Multi-Diminsional Nonlocal Operators Boris N. KHOROMSKIJ (joint work with Wolfgang Hackbusch)}

The class of $\mathcal{H}$-matrices allows an approximate matrix arithmetic with almost linear complexity. The combination of the hierarchical and tensor-product format offers the opportunity for efficient data-sparse representation of integral operators and the inverse of elliptic operators in higher dimensions (cf. [2], [1], [3]). In the present talk, we discuss the $\mathcal{H}$-matrix techniques combined with the Kronecker tensor-product approximation to represent integral operators as well as certain functions $\mathcal{F}(A)$ of a discrete elliptic operator $A$ in a hypercube $(0,1)^{d} \in \mathbb{R}^{d}$ in the case of a high spatial dimension $d$. In particular, we approximate the functions $A^{-1}$ and $\operatorname{sign}(A)$ of a finite difference discretisations $A \in \mathbb{R}^{N \times N}$ with rather general location of the spectrum. The asymptotic complexity of our data-sparse representations can be estimated by $\mathcal{O}\left(n^{p} \log ^{q} n\right), p=1,2$, with $q$ independent of $d$, where $n=N^{1 / d}$ is the dimension of the discrete problem in one space direction. 


\section{REFERENCES}

[1] I. P. Gavrilyuk, W. Hackbusch, and B. N. Khoromskij: Tensor-product approximation to elliptic and parabolic solution operators in higher dimensions. Preprint 83, Max-PlanckInstitut für Mathematik in den Naturwissenschaften, Leipzig 2003; Computing (to appear).

[2] W. Hackbusch, B.N. Khoromskij, and E. Tyrtyshnikov: Hierarchical Kronecker tensorproduct approximation, Preprint 35, Max-Planck-Institut für Mathematik in den Naturwissenschaften, Leipzig, 2003 (submitted).

[3] W. Hackbusch and B.N. Khoromskij. Hierarchical Kronecker tensor-product approximation to a class of nonlocal operators in higher dimensions. Preprint 16, MPI MIS, Leipzig 2004 (submitted).

\section{Integral equations and numerical methods for inverse scattering problems}

\section{RAINER KRESS}

This presentation provides a survey on some recent developments in the numerical solution of time-harmonic inverse obstacle scattering problems. After formulating the inverse problem, the issue of uniqueness, that is, identifyability will be addressed. The uniqueness question is of its own mathematical interest and also interrelates with some of the more recently developed reconstruction algorithms. By considering one or two of its representatives the basic ideas of three groups of methods will be outlined, namely decomposition methods, iterative methods and sampling and probe methods.

Consider the scattering of a time-harmonic acoustic plane wave $u^{i}$ from an impenetrable scatterer described by a bounded domain $D$ in $\Re^{3}$ either with a sound-soft or an impedance boundary condition. The inverse obstacle scattering problem consists of finding the shape and location of $D$ from the knowledge of the far field pattern $u_{\infty}$ of the scattered wave $u^{s}$ for one or several incident plane waves. The corresponding uniqueness result due to Kirsch and Kress [15] (see also [4]) confirms that the domain $D$ and the boundary condition are uniquely determined by the far field pattern for infinitely many incident plane waves. The main idea of the proof is to exploit the fact that for scattering of a point source the scattered wave develops singularities when the source and observation points approach the boundary. Uniqueness for one incident plane wave remains a challenging open problem. Partial results were recently obtained for scattering from polyhedral scatterers [1].

Decomposition methods, in principle, separate the inverse problem into an illposed linear problem to reconstruct the scattered wave $u^{s}$ from its far field pattern $u_{\infty}$ and a nonlinear problem for the subsequent determination of the boundary $\partial D$ of the scatterer from the boundary condition. These methods do not require the solution of the forward problem and some of them perform well without a priori information on the geometry of the obstacle. A typical representative of this approach is the potential method of Kirsch and Kress (see [6, 14]).

Iteration methods interpret the inverse obstacle scattering problem as a nonlinear ill-posed operator equation $A(\partial D)=u_{\infty}$ and apply iterative schemes such 
as regularized Newton type, Landweber or conjugate gradient methods for its solution. Here, $A$ denotes the operator that, for a fixed incident field, maps the boundary $\partial D$ of the scatterer onto the far field pattern of the scattered wave. The theoretical foundation for this approach requires to establish the differentiability of the operator $A$ with respect to the boundary and to explicitly characterize the derivative. For the sound-soft and the impedance boundary condition this was done, among others, by Kirsch [12] and by Hettlich [8] via variational methods, by Potthast $[18,19]$ via integral equation methods and by Kress and Päivärinta [17] and by Haddar and Kress [7] via factorization formulas. For details on the numerical implementation we refer to $[4,5,9,12,16]$. The numerical examples provide amble evidence that iterative methods yield very good reconstructions. However, they require the solution of the corresponding forward problem in each iteration step and a priori information on the geometry of the obstacle. Furthermore, although progress has been made through the work of Hohage [10] and Potthast [21], the convergence issue is not yet satisfactorily settled.

The main idea of the more recently developed so-called sampling and probe methods is to develop a criterium in terms of the behaviour of some ill-posed linear integral equation that decides on whether a point $z$ lies inside or outside the scatterer $D$. Then the criterium is evaluated numerically for a grid of points to visualize the unknown scatterer. As opposed to the two previous types of methods that, in principle, only need the far field pattern for one incident direction, the sampling and probe methods need the far field pattern for all incident and observation directions. However, as their main advantage they perform extremely well without any a priori information on the geometry. The linear sampling method as developed by Colton and Kirsch [3] has as its central piece the far field operator $F: L^{2}(\Omega) \rightarrow L^{2}(\Omega)$ on the unit sphere $\Omega$. This operator is defined as an integral operator with the kernel given by the far field pattern $u_{\infty}(\hat{x}, d)$ for all observation directions $\hat{x} \in \Omega$ and all incident directions $d$. With the explicitly available far field pattern $w_{\infty}^{i}(\cdot, z)$ of the field of an point source located at the point $z$ the linear sampling method is based on the ill-posed linear integral equation $\mathrm{Fg}(\cdot, z)=w_{\infty}^{i}(\cdot, z)$. Although, this integral equation, in general, is not solvable, it can be approximately solved in the sense that for every $\varepsilon>0$, and $z \in D$ there exists $g(\cdot, z) \in L^{2}(\Omega)$ such that $\left\|F g(\cdot, z)-w_{\infty}^{i}(\cdot, z)\right\|_{L^{2}(\Omega)} \leq \varepsilon$ and $\|g(\cdot, z)\|_{L^{2}(\Omega)} \rightarrow \infty$ as $z \rightarrow \partial D$. In the numerical implementation the far field equation is solved by Tikhonov regularization via Morozov's discrepancy principle and then $\partial D$ is visualized through the points $z$ where $\|g(\cdot, z)\|_{L^{2}(\Omega)}$ becomes large. A remaining gap in the theoretical foundation of the linear sampling method, namely, the question why the implementation via Tikhonov and Morozov actually picks the approximation $g$ that is predicted by the above theoretical result was closed by Arens [2].

The factorization method may be considered as a variation of the linear sampling method in the sense that it replaces $F$ in the far field equation by $\left(F^{*} F\right)^{1 / 4}$, that is, it is based on the equation $\left(F^{*} F\right)^{1 / 4} g(\cdot, z)=w_{\infty}^{i}(\cdot, z)$. As shown in a pioneering paper by Kirsch [13] this equation is more satisfying, since it is solvable 
if and only if $z \in D$. The numerical implementation of the factorization method is similar to that of the linear sampling method. The procedure is known as factorization method, since it relies on a factorization of the far field operator. The linear sampling method and the factorization method may be viewed as dual to the uniqueness proof of Kirsch and Kress, since, in principle, their foundation is based on letting source points approach the boundary from inside of $D$ whereas in the uniqueness proof the source points approch the boundary from outside of $D$. The latter idea is mimiced in the point source and singular source methods of Potthast [20] and the probe method of Ikehata [11].

\section{REFERENCES}

[1] Alessandrini, G. and Rondi, L.: Determing a sound-soft polyhedral scatterer by a single far-field measurement. Proc. Amer. Math. Soc. (to appear).

[2] Arens, T.: Why linear sampling works. Inverse Problems 20, 163-173 (2004).

[3] Colton, D. and Kirsch, A.: A simple method for solving inverse scattering problems in the resonance region, Inverse Problems 12 383-393, (1996).

[4] Colton, D. and Kress, R.: Inverse Acoustic and acoustic Scattering Theory. 2nd. ed. Springer, Berlin 1998.

[5] Farhat, C., Tezaur, R., and Djellouli: On the solution of three-dimensional inverse obstacle acoustic scattering problems by a regularized Newton method. Inverse Problems 18, 12291246 (2002).

[6] Haas, M., Rieger, W., Rucker, W., and Lehner, G.: Inverse 3D acoustic and electromagnetic obstacle scattering by iterative adaption. In: Inverse Problems of Wave Propagation and Diffraction (Chavent and Sabatier, eds). Springer-Verlag, Heidelberg 1997.

[7] Haddar, H. and Kress. R.: On the Frechet derivative for obstacle scattering with an impedance boundary condition. SIAM J. Appl. Math. (to appear).

[8] Hettlich, F.: Fréchet derivatives in inverse obstacle scattering. Inverse Problems 11, 371-382 (1995).

[9] Hohage, T.: Logarithmic convergence rates of the iteratively regularized Gauss-Newton method for an inverse potential and an inverse scattering problem. Inverse Problems 13, 1279-1299 (1997).

[10] Hohage, T.: Iterative Methods in Inverse Obstacle Scattering: Regularization Theory of Linear and Nonlinear Exponentially Ill-Posed Problems. Dissertation, Linz 1999.

[11] Ikehata, M.: Reconstruction of an obstacle from the scattering amplitude at a fixed frequency. Inverse Problems 14, 949-954 (1998).

[12] Kirsch, A.: The domain derivative and two applications in inverse scattering theory. Inverse Problems 9, 81-96 (1993).

[13] Kirsch, A.: Characterization of the shape of a scattering obstacle using the spectral data of the far field operator. Inverse Problems 14, 1489-151 (1998).

[14] Kirsch, A., and Kress, R.: An optimization method in inverse acoustic scattering. In: Boundary elements IX, Vol 3. Fluid Flow and Potential Applications (Brebbia et al, eds.) pp. 3-18, Springer-Verlag, Berlin Heidelberg New York, 1987.

[15] Kirsch, A., and Kress, R.: Uniqueness in inverse obstacle scattering. Inverse Problems 9, 285-299 (1993).

[16] Kress, R.: Integral equation methods in inverse acoustic and acoustic scattering. In: Boundary Integral Formulations for Inverse Analysis, (Ingham, Wrobel, eds.), pp 67-92, Computational Mechanics Publications, Southampton 1997.

[17] Kress, R. and Päivärinta, L.: On the far field in obstacle scattering. SIAM J. Appl. Math. 59, 1413-1426 (1999).

[18] Potthast, R.: Fréchet differentiability of boundary integral operators in inverse acoustic scattering. Inverse Problems 10, 431-447 (1994). 
[19] Potthast, R.: Fréchet differentiability of the solution to the acoustic Neumann scattering problem with respect to the domain. Jour. on Inverse and Ill-posed Problems 4, 67-84 (1996).

[20] Potthast, R.: A point-source method for inverse acoustic and electromagnetic obstacle scattering problems. IMA Journal of Appl. Math. 61, 119-140 (1998).

[21] Potthast, R.: On the convergence of a new Newton-type method in inverse scattering. Inverse Problems 17, 1419-1434 (2001).

\section{A hypersingular integral equation arising in a waveguide scattering problem \\ Giovanni Monegato \\ (joint work with Renato Orta)}

In this work we study the electromagnetic scattering problem associated with a T-junction between two rectangular waveguides. This junction is composed by an infinite (primary) waveguide

$$
\Omega_{1}=\left\{(x, y, z) \in R^{3}: 0<x<a, 0<y<b,-\infty<z<\infty\right\}
$$

and by a semi-infinite (secondary) waveguide

$$
\Omega_{2}=\left\{(x, y, z) \in R^{3}:-\infty<x<0,0<y<b, 0<z<a^{\prime}\right\} .
$$

They are coupled through the common aperture

$$
A=\left\{(x, y, z) \in R^{3}: x=0,0<y<b, 0<z<a^{\prime}\right\}
$$

where in general $a^{\prime} \neq a$.

Notice that in this particular configuration the two waveguides have the same height $b$. This assumption will reduce to 2 the dimension of the problem. The case of two waveguides with different heights appears more difficult and will be considered in a future paper.

We assume that the electromagnetic field varies harmonically (sinusoidally) with time, with angular frequency $\omega$ and time factor $e^{j \omega t}$. The dielectric medium is assumed to be homogeneous and isotropic. The magnetic permeability is denoted by $\mu$ and the electric permittivity by $\epsilon$. We assume $\epsilon=\epsilon^{\prime}-j \epsilon^{\prime \prime}$, with $\epsilon^{\prime}, \epsilon^{\prime \prime}>0$ and $\epsilon^{\prime \prime}<<\epsilon^{\prime}$, which means that the medium is weakly dissipative.

If in the structure we have a magnetic current density $J_{m}$ and an electric current density $J_{e}$, then the total electric and magnetic fields, denoted by $E$ and $H$, respectively, satisfy Maxwell equations in the domain $\Omega$ given by the union of $\Omega_{1}, \Omega_{2}$ and the aperture $A$. The boundary of this domain will be denoted by $\Gamma$. In the problem we consider we take

$$
J_{e}=0 .
$$

However, at a given arbitrary section $z=-d, d>0$, of the primary waveguide we introduce a magnetic source of the type

$$
J_{m}=2 \sqrt{\frac{2}{a b}} V_{0}^{i} \sin \left(\frac{\pi}{a} x\right) \delta(z+d) \hat{x},
$$


producing the incident electric field

$$
E^{i}(x, z)=\sqrt{\frac{2}{a b}} V_{0}^{i} \sin \left(\frac{\pi}{a} x\right) e^{-j k_{z 1}(z+d)} \hat{y},
$$

corresponding to the fundamental mode (eigenfunction) in the primary waveguide, where $V_{0}^{i}$ is a given constant and $k_{z 1}=\sqrt{k^{2}-\left(\frac{\pi}{a}\right)^{2}}$. Here and in the following, $\hat{x}, \hat{y}, \hat{z}$ denote the cartesian unit vectors, and the square root is always determined by taking the root with negative imaginary part. The quantity $k=\omega \sqrt{\epsilon \mu}$ is the medium wavenumber, which has the form $k=k^{\prime}-j k^{\prime \prime}$, with $k^{\prime}, k^{\prime \prime}>0\left(k^{\prime \prime}<<k^{\prime}\right)$.

The following incident magnetic field is associated with the above incident electric field:

$$
\begin{aligned}
& H^{i}(x, z)=-V_{0}^{i} \frac{k_{z 1}}{k} Y_{0} \sqrt{\frac{2}{a b}} \sin \left(\frac{\pi}{a} x\right) e^{-j k_{z 1}(z+d)} \hat{x} \\
& -j V_{0}^{i} \frac{\pi}{k a} Y_{0} \sqrt{\frac{2}{a b}} \cos \left(\frac{\pi}{a} x\right) e^{-j k_{z 1}(z+d)} \hat{z},
\end{aligned}
$$

where $Y_{0}=\sqrt{\frac{\epsilon}{\mu}}$.

The total fields $E, H$ are the sum of the incident fields and the corresponding scattered ones.

The junction walls are assumed to be a perfect electric conductor. Therefore the tangential component of the electrical field must vanish on them, that is,

$$
\hat{n} \times E=0 \quad \text { on } \Gamma,
$$

$\hat{n}$ being the normal unit vector. Notice that this assumption also implies

$$
\hat{n} \cdot H=0 \quad \text { on } \Gamma \text {. }
$$

Finally, $E$ and $H$ must satisfy Sommerfeld radiation condition.

At this point it is of key importance to notice that because both the geometry of the structure and the incident field are invariant with respect to the $y$ coordinate, the fields $E=\left(E_{x}, E_{y}, E_{z}\right)^{T}$ and $H=\left(H_{x}, H_{y}, H_{z}\right)^{T}$ are constant with respect to $y$. This, together with conditions (5) and (6), implies

$$
E_{x}=E_{z}=H_{y}=0 \quad \text { in } \Omega .
$$

Therefore, the only unknown components of $E$ and $H$ are $E_{y}, H_{x}, H_{z}$.

Taking into account this last result, and denoting by $\Omega_{x z}$ the section $y=$ constant of the domain $\Omega$, and by $\Gamma_{x z}$ its boundary, a standard calculation reduces Maxwell equations and their boundary conditions to the simpler scalar two-dimensional form

$$
\begin{aligned}
& \begin{cases}\left(\nabla_{x z}^{2}+k^{2}\right) E_{y}=\frac{\partial J_{m_{x}}}{\partial z}, & \text { in } \Omega_{x z} \\
E_{y}=0, & \text { on } \Gamma_{x z} \\
\lim _{x \rightarrow-\infty} E_{y}(x, z)=\lim _{z \rightarrow \pm \infty} E_{y}(x, z)=0 ; & \end{cases} \\
& \left\{\begin{array}{l}
H_{x}=\frac{1}{j \omega \mu}\left[\frac{\partial E_{y}}{\partial z}-J_{m_{x}}\right], \\
H_{z}=-\frac{1}{j \omega \mu}\left[\frac{\partial E_{y}}{\partial x}+J_{m_{z}}\right] .
\end{array}\right.
\end{aligned}
$$


¿From the application point of view, the goal is the computation of the so-called scattering matrix, that is, of the (normalized) Fourier coefficients of the scattered electric field with respect to the structure modes. There is no interest in knowing the pointwise values of the scattered field.

To solve the problem we perform a domain decomposition of $\Omega_{x z}$ into the two separate waveguides. This because much is known about Helmholtz equation in a single rectangular waveguide.

To this end, by invoking a well-known surface equivalence principle in electromagnetic theory, we close the aperture $A$ of the T-junction by inserting a thin metallic wall, and on the two faces of it we introduce equivalent magnetic currents: $+J_{m}^{e q}=-M(z) \delta\left(x-0^{+}\right) \hat{z}, 0 \leq z \leq a^{\prime}$, on the primary waveguide side and $-J_{m}^{e q}=M(z) \delta\left(x-0^{-}\right) \hat{z}$ on the secondary one. This unknown fictitious current is introduced to make the new decoupled problem equivalent to the original one.

In each waveguide the problem reduces to a non-homogeneous Helmholtz equation, with homogeneous boundary conditions, for the scattered electric field. An integral representation for this field is then obtained, from which the corresponding expression for the scattered magnetic field follows.

By imposing the continuity of the tangential component $H_{z}$ of the total magnetic fields generated in the two separated waveguides at their interface $A$, we obtain a hypersingular integral equation, defined on the interval $\left(0, a^{\prime}\right)$, whose solution yields the unknown fictitious magnetic current $M(z)$. From this, the scattering matrix of the junction is easily computed.

This integral equation has two kernels given in terms of series expansions. Our analysis will determine all singular components of the kernels and will show that besides the standard second order hypersingularity, one of the kernel also has a fixed-point second order hypersingularity at each endpoint of the interval of integration.

The equation is finally solved by means of a Galerkin method, whose implementation is performed quite efficiently. Indeed a fast and accurate procedure is proposed to compute the Galerkin matrix elements. The overall numerical method is very fast and accurate.

\section{REFERENCES}

[1] R.F. Harrington, Time-Harmonic Electromagnetic Fields, McGraw-Hill, New York, 1961.

[2] E.D. Sharp, An exact calculation for T-junction of rectangular waveguides having arbitrary cross sections, IEEE Trans. Microwave Theory Tech., vol. 15, 1967, pp.109-116.

[3] J.M. Rebollar, J. Esteban, J.E. Page, Fullwave analysis of three and four-port rectangular waveguide junctions, IEEE Trans. Microwave Theory Tech., vol. 24, 1984, pp.256-263.

[4] X.P. Liang, K.A. Zaki, A.E. Atia, A rigorous three plane mode-matching technique for characterizing waveguide T-junctions and its application in multiplexer design, IEEE Trans. Microwave Theory Tech., vol. 39, 1991, pp.2138-2147.

[5] T. Sieeverding, F. Arndt, Field theoretic CAD of open or aperture matched T-junction coupled rectangular waveguide structures, IEEE Trans. Microwave Theory Tech., vol. 40, 1992, pp.353-362.

[6] G.W. Hanson, A.B. Yakovlev, Operator Theory for Electromagnetics, Springer-Verlag, New York, 2002. 


\section{On nonlocal electrostatics \\ Sergej RJasanow}

\section{Introduction}

In recent years a significant interest has been focused on the determination of electrostatic potentials of large biomolecules such as, e.g., microtubule and ribosomes [1]. However, the standard continuum approach ultimately becomes inaccurate when used to determine electrostatic properties on atomic scales [3], as it is featureless, i.e., the correlation between solvent arrangements and the geometrical structure of biomolecular assemblies is not taken into account.

In the recent paper [2] we proposed a novel formulation of nonlocal electrostatics allowing numerical solutions for the nontrivial molecular geometries arising in the applications mentioned before. For many relevant models, the dielectric function of the medium can be expressed as the Green's function of the Yukawa operator. In this case, a system of coupled PDE's can be used as a mathematical model of the problem.

\section{Nonlocal electrostatics}

Let $\Omega \subset \mathbb{R}^{3}$ be a bounded, simply connected domain having piecewise smooth boundary $\Gamma=\partial \Omega$ as it is shown in Fig 1 . The outward unit normal vector at $x \in \Gamma$ will be denoted by $n_{x}$. There are $N_{q}$ point charges inside of the domain $\Omega$. Thus the spatial charge density $\rho$ in $\Omega$ can be given in the form of point measure

$$
\rho(x)=\sum_{j=1}^{N_{q}} q_{j} \delta\left(x-x_{j}\right), x_{j} \in \Omega, j=1, \ldots, N_{q}
$$

The complement domain $\Omega^{C}=\mathbb{R}^{3} \backslash \bar{\Omega}$ is filled with water.

The problem is to determine the electric field $\vec{E}(x)$ for all $x \in \mathbb{R}^{3}$, i.e. inside and outside of the domain $\Omega$. The electric field is described by a system of Maxwell equations which reads as

(2) $\left\{\begin{array}{l}\operatorname{div} \vec{D}(x)=\rho(x) \\ \operatorname{rot} \vec{E}(x)=0\end{array} \quad, x \in \Omega,\left\{\begin{array}{l}\operatorname{div} \vec{D}(x)=0 \\ \operatorname{rot} \vec{E}(x)=0\end{array}, x \in \Omega^{C}=\mathbb{R}^{3} \backslash \bar{\Omega}\right.\right.$.

where the spatial density $\rho$ is given in (1). On $\Gamma$ the usual interface conditions on $\Gamma$ for the vector fields $\vec{E}$ and $\vec{D}$ are formulated

(3) $\left(\gamma_{0}^{e x t} \vec{D}(x)-\gamma_{0}^{i n t} \vec{D}(x), n_{x}\right)=0,\left(\gamma_{0}^{e x t} \vec{E}(x)-\gamma_{0}^{i n t} \vec{E}(x)\right) \times n_{x}=0$,

where $\gamma_{0}^{\text {int }}$ and $\gamma_{0}^{\text {ext }}$ are the interior and exterior Dirichlet trace operators. Thus the normal component of the vector field $\vec{D}$ and the tangential component of the vector field $\vec{E}$ are continuous on the boundary $\Gamma$. The Maxwell equations (2) are completed with the usual, local material relationship in $\Omega$

$$
\vec{D}(x)=\varepsilon_{0} \varepsilon_{\Omega} \vec{E}(x), x \in \Omega .
$$




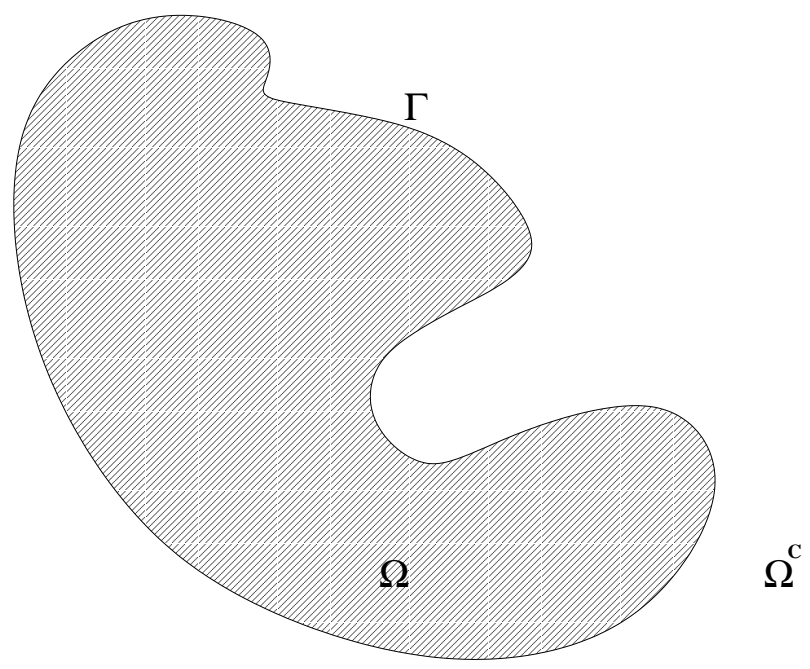

Figure 1. Computational domain $\Omega$

The material relationship in $\Omega^{C}$ is nonlocal and can be written as follows

$$
\vec{D}(x)=\varepsilon_{0} \varepsilon_{\infty} \vec{E}(x)+\gamma \int_{\Omega^{C}} u_{2}^{*}(x, y) \vec{E}(y) d y, x \in \Omega^{C} .
$$

In (5), the function $u_{2}^{*}: \mathbb{R}^{3} \times \mathbb{R}^{3} \rightarrow \mathbb{R}$ denotes the fundamental solution of the Yukawa equation

$$
u_{2}^{*}(x, y)=\frac{1}{4 \pi} \frac{e^{-\kappa|x-y|}}{|x-y|}
$$

Thus the mathematical model of non-local electrostatic consists of the Maxwell equations (2), interface conditions (3) together with the material relationships (4) and (5).

\section{System OF PDE's}

In this subsection we consider a system of partial differential equations for our problem using the scalar functions $u: \Omega \rightarrow \mathbb{R}$ and $v: \Omega^{C} \rightarrow \mathbb{R}$ with

$$
\vec{E}(x)=-\operatorname{grad} u(x), x \in \Omega, \vec{E}(x)=-\operatorname{grad} v(x), x \in \Omega^{C}
$$

as well as a scalar harmonic functions $w: \Omega_{C} \rightarrow \mathbb{R}$. Instead of the Maxwell equations (2) we consider the following system of PDE's for the functions $u, v$ and 
$w$

$$
\begin{aligned}
-\varepsilon_{0} \varepsilon_{\Omega} \Delta u(x) & =\rho(x), x \in \Omega, \\
-\Delta w(x) & =0, x \in \Omega^{C}, \\
-\varepsilon_{0} \varepsilon_{\infty}\left(\Delta v(x)-\left(\kappa^{\prime}\right)^{2} v(x)\right) & =\kappa^{2}\left(w+w^{*}\right)(x), x \in \Omega^{C},
\end{aligned}
$$

where $\kappa^{\prime}=\sqrt{\kappa^{2}+\gamma / \varepsilon_{0}}$ and the function $w^{*}: \mathbb{R}^{3} \rightarrow \mathbb{R}$ is defined as follows

$$
w^{*}(x)=\frac{1}{4 \pi} \sum_{j=1}^{N_{q}} \frac{q_{j}}{\left|x-x_{j}\right|} .
$$

The system (8) is subjected to the following interface conditions of $\Gamma$

$$
\begin{aligned}
-\gamma_{1}^{e x t}\left(w(x)+w^{*}(x)\right)+\varepsilon_{0} \varepsilon \gamma_{1}^{i n t} u(x) & =0, \\
\gamma_{0}^{e x t} v(x)-\gamma_{0}^{i n t} u(x) & =0, \\
\gamma_{1}^{e x t} v(x)-\gamma_{1}^{i n t} u(x) & =0 .
\end{aligned}
$$

The system (8) with the interface conditions (12) is not equivalent to the previous model of nonlocal electrostatics because of the continuity of the electric field $\vec{E}$ on the boundary $\Gamma$ forced by the third interface condition. However the Maxwell equations, local material relationship in $\Omega$ as well as the nonlocal material relationship in $\Omega^{C}$ are formally fulfilled. The system (8) contains three PDE's with constant coefficients and therefore can be solved using boundary element methods. In Table 1 . the numerical values of the solvation enthalpy (third column) are compared with measurements (fourth column) for four simple ions.

\begin{tabular}{|r|c|c|c|}
\hline Ammonium & $\mathrm{NH}_{4}^{+}$ & -83.6 & -79.0 \\
Methanolat & $\mathrm{CH}_{3} \mathrm{O}^{-}$ & -78.6 & -95.0 \\
Methylammonium & $\mathrm{CH}_{3} \mathrm{NH}_{3}^{+}$ & -68.5 & -70.0 \\
Anilinium & $\mathrm{C}_{5} \mathrm{H}_{5} \mathrm{NH}^{+}$ & -59.3 & -59.0 \\
\hline
\end{tabular}

Table 1. Computational results and measurements

\section{REFERENCES}

[1] N. A. Baker, D. Sept, S. Joseph, M. J. Holst, and J. A. McCammon. In Proc. Nat. Acad. Sci., number 98, page 10037, 2001. USA.

[2] A. Hildebrandt, R. Blossey, S. Rjasanow, O. Kohlbacher, and H.-P. Lenhof. A novel formulation of nonlocal electrostatics. Phys. Rev. Let., 2004. to appear.

[3] T. Simonson. Curr. Op. Struct. Biol, 11:243, 2001. 


\title{
Stabilisation of the acoustic single layer potential on non-smooth domains
}

\author{
Stefan A. Sauter \\ (joint work with Annalisa Buffa)
}

\section{INTRODUCTION}

In our presentation, we will address problems related to the discretization of boundary integral equations for the Helmholtz problem outside of a reflecting obstacle $\Omega^{-}$, where $\Omega^{-} \subset \mathbb{R}^{3}$ is a bounded Lipschitz domain. Let $\Omega^{+}=\mathbb{R}^{3} \backslash \bar{\Omega}^{-}$ and $\mathcal{L}_{k}:=-\Delta-k^{2}$. We consider the problem: Find $u^{+} \in H_{l o c}^{1}\left(\Omega^{+}\right)$such that the Helmholtz problem

$$
\begin{aligned}
& \mathcal{L}_{k} u^{+}=0 \quad \text { in } \Omega^{+}, \\
& u^{+}=g \quad \text { on } \Gamma:=\partial \Omega^{-} \text {, } \\
& \left.|u(\mathbf{x})| \leq C\|\mathbf{x}\|^{-1}\right\} \text { for }\|\mathbf{x}\| \rightarrow \infty \\
& \left.\left|\frac{\partial u}{\partial r}-\mathrm{i} k u\right| \leq C\|\mathbf{x}\|^{-2}\right\} \text { for }\|\mathbf{x}\| \rightarrow \infty
\end{aligned}
$$

is satisfied in a weak sense (cf. [25]).

Our goal is to solve these equations by the method of integral equations. A potential ansatz leads to a boundary integral equation on $\Gamma$ for the unknown density $\varphi$ which is of the form $R_{k} \varphi=g$. Here, $R_{k}$ is the trace $V_{k}$ of the single layer potential associated to $\mathcal{L}_{k}$ on $\Gamma$ or a stabilized version of it. We will consider the Galerkin boundary element method for its discretization. It is well known that the $V_{k}$ is not invertible on a countable set of frequencies $k$ (see, e.g., [10]) and we will introduce a class of stabilizations such that the boundary integral equation is well posed for all frequencies $k>0$.

It is well known (see, e.g., [3]) that finite element discretizations for the Helmholtz problem suffer from the pollution effect, i.e., the constants in the Galerkin error estimates deteriorates to infinity with increasing wave number $k>0$. Hence, the question arises whether this pollution effect is possibly reduced by solving the boundary integral equation for the Helmholtz problem via the Galerkin boundary element method.

We will analyze the effect of the frequency $k$ in the error of the Galerkin boundary element solution. First, we will introduce a family of stabilizations to eliminate the forbidden frequencies and then analyze the pollution effect for the boundary element discretization of the corresponding stabilized variational equations.

There exist various approaches in the literature to stabilize the single layer potential for the Helmholtz problem (see [9], [10], [19], [22, Sec. 6.4]). Existence and uniqueness have been proved and, for the Galerkin boundary element method, it could be shown that the convergence is quasi-optimal provided the step size is "sufficiently small". More precisely, the threshold for the maximal step size such that the Galerkin discretization is stable strongly depends on the wave number 
and the "constant" in the quasi-optimality error estimate deteriorates to infinity as the wave number increases.

Consequently, in order to compare the different approaches from the viewpoint of numerical efficiency the following questions have to be addressed:

(1) How does the threshold for the stability of the Galerkin discretization quantitatively depend on the wave number?

(2) How does the Galerkin error quantitatively depend on the mesh width and the wave number?

(3) Can the stabilization approach be implemented efficiently in a boundary element code? What is the computational complexity?

These questions have been discussed for the Brakhage-Werner stabilization in [16] (see also [23]). We will address these questions for a general stabilization approach for the acoustic single layer operator $V_{k}$.

\section{RESUlts}

We will briefly summarize the results. All details can be found in [8].

a) The stabilized acoustic single layer potential, on the continuous level, admits a unique solution which depends continuously on the data for general Lipschitz surfaces. This can be considered as an advantage compared to the Brakhage-Werner stabilization, where the question of existence and uniqueness is open for general Lipschitz surfaces and even for piecewise smooth surfaces.

b) The Galerkin method converges for "sufficiently small" step size on general triangulated surfaces with optimal rate.

We have analyzed quantitatively the dependence of the constants entering the stability and convergence estimates in the case that the surface is the unit sphere in $\mathbb{R}^{3}$. For this case, we obtain:

c) The condition for the stability of Galerkin method (related to the condition "the step size has to be sufficiently small") is more restrictive as for the stabilization in the Brakhage-Werner approach.

d) The constants in the Galerkin error estimates depend on the wave-number in a more critical way as for the Brakhage-Werner stabilization.

\section{REFERENCES}

[1] M. Abramowitz and I. A. Stegun. Applied Mathematics Series 55. National Bureau of Standards, U.S. Department of Commerce, 1972.

[2] S. Amini, On the choice of the coupling parameter in boundary integral formulations of the exterior acoustic problem, Appl. Anal., 35 (1990), pp. 75-92.

[3] I. Babuška and S. Sauter. Is the pollution effect of the FEM avoidable for the Helmholtz equation considering high wave numbers? SIAM Rev., 42(3):451-484 (electronic), 2000. Reprint of SIAM J. Numer. Anal. 34 (1997), no. 6, 2392-2423. 
[4] H. Brakhage and P. Werner. Über das Dirichletsche Außenraumproblem für die Helmholtzsche Schwingungsgleichung. Arch. der Math., 16: 325-329, 1965.

[5] A. Brandt and A.A. Lubrecht. Multilevel Matrix Multiplication and Fast Solutions of Integral Equations. J. Comp. Physics, 90: 348-370, 1990.

[6] A. Buffa and P. Ciarlet, Jr. On traces for functional spaces related to Maxwell's equations. Part I: An integration by parts formula in Lipschitz polyhedra. Math. Meth. Appl. Sci., 21(1):9-30, 2001.

[7] A. Buffa and R. Hiptmair. A coercive combined field integral equation for electromagnetic scattering. Technical Report 1325, IMATI-CNR, 2003. To appear in SIAM J. Numer. Anal.

[8] A. Buffa and S. A. Sauter. Stabilisation of the acoustic single layer potential on non-smooth domains. Preprint Nr. 19-2003, Univ. Zürich, 2003.

[9] A. Burton and G. Miller. The application of integral methods for the numerical solution of boundary value problems. Proc. R. Soc. London, A232: 201-210, 1971.

[10] D. Colton and R. Kress. Inverse acoustic and electromagnetic scattering theory, volume 93 of Applied Mathematical Sciences. Springer-Verlag, Berlin, 1992.

[11] M. Costabel. Boundary integral operators on Lipschitz domains: elementary results. SIAM J. Math. Anal., 19(3):613-626, 1988.

[12] M. Dauge. Elliptic boundary value problems on corner domains. Lecture Notes in Mathematics. Springer Verlag, Berlin, 1988.

[13] L. Demkowicz. Asymptotic convergence in finite and boundary element methods: Part 2, The LBB constant for rigid and elastic scattering problems. Comput. Math. Appl., 28(6):93109, 1994.

[14] Y. V. Egorov, A. I. Komech and M. A. Shubin. Elements of the Modern Theory of Partial Differential Equations. Springer Verlag, 1999.

[15] S. Erichsen and S.A. Sauter. Efficient automatic quadrature in 3-d Galerkin BEM. Comp. Meth. Appl. Mech. Eng., 157:215-224, 1998.

[16] K. Giebermann. Schnelle Summationsverfahren zur numerischen Lösung von Integralgleichungen für Streuprobleme im $\mathbb{R}^{3}$, PhD thesis, Universität Karlsruhe, Germany, 1997.

[17] I.G. Graham, W. Hackbusch and S.A. Sauter.Discrete boundary element methods on general meshes in 3D. Numer. Math., 86:103-137, 2000.

[18] W. Hackbusch. Elliptic Differential Equations. Springer Verlag, 1992.

[19] R. Hiptmair and A. Buffa. Coercive Combined Field Integral Equations. Report No. 2003-08, SAM ETHZ Zürich, 2003.

[20] D. Jerison and C.E. Kenig. The inhomogeneous Dirichlet problem in Lipschitz domains. J. Funct. Anal., 130:161-219, 1995.

[21] R. Kress. Minimizing the Condition Number of Boundary Integral Operators in Acoustics and Electromagnetic Scattering. Q. Jl. Mech. appl. Math., 38:323-341, 1985.

[22] R. Kress. Linear Integral Equations, Springer-Verlag, Berlin, 1989.

[23] R. Kress and W. T. Spassov. On the condition number of boundary integral operators for the exterior Dirichlet problem for the Helmholtz equation. Numer. Math., 42(1):77-95, 1983.

[24] C. Lage, G. Schmidlin and C. Schwab. Rapid solution of first kind boundary integral equations in $\mathbb{R}^{3}$. Engineering Analysis with Boundary Elements, 27(5) :469-490, 2003.

[25] R. Leis. Initial Boundary Value Problems in Mathematical Physics, Teubner, Wiley \& Sons, Stuttgart, Chichester, 1986.

[26] J.-L. Lions and E. Magenes. Non-homogeneous Boundary value Problems and Applications, Springer-Verlag, 1970.

[27] S. Mikhlin and S. Prössdorf. Singular Integral Operators. Springer-Verlag, Heidelberg, 1986.

[28] J. C. Nédélec. Acoustic and Electromagnetic Equations. Springer, New York, 2001.

[29] N. Ortner and P. Wagner. A Survey on Explicit Representation Formulae for Fundamental Solutions of Linear Partial Differential Operators. Acta Applicandae Mathematicae, 47:101124, 1997.

[30] O. Panich. On the question of the solvability of the exterior boundary-value problems for the wave equation and Maxwell's equation. Russ. Math. Surveys, 20:221-226, 1965. 
[31] G. Schmidt and B. N. Khoromskij. Boundary integral equations for the biharmonic Dirichlet problem on nonsmooth domains. J. Integral Equations Appl., 11(2):217-253, 1999.

[32] K. Yosida, Functional Analysis, Classics in Mathematics, Springer, 1995. Reprint of the 1980 edition.

\section{Adaptive Wavelet Based Fast Solution of BEM REINHOLD SCHNEIDER \\ (joint work with Wolfgang Dahmen, Helmut Harbrecht)}

During the past years adaptive wavelet methods based on best $N$-term approximation have been introduced by Cohen, Dahmen, DeVore. Like Fast Multipole Method, Panel Clustering etc. which been developed to reduce the complexity to an optimal or almost optimal rate, it is known that the Galerkin discretization using wavelet bases yields immediately a quasi-sparse representation. Combining this matrix compression, with wavelets adaptive approximation of the solution gives a powerful instrument for solving integral equations. We present an adaptive wavelet scheme which is observed not compromising the acurracy of the full Galerkin scheme on the full (quasi-) uniform grid.

\section{REFERENCES}

[1] A. Barinka, W. Dahmen and R. Schneider: Fast computation of adaptive wavelet expansions, IGPM Report \#244, RWTH Aachen, July 2004, submitted to Numer. Math.

[2] A. Cohen, W. Dahmen, R., DeVore Adaptive wavelet methods for elliptic equations - convergence rates, Math. Comp. 70, p. 27-75, (2001)

[3] H. Harbrecht and R. Schneider, Wavelet Based Fast Solution of Boundary Integral Equations, in Proc. of the Internat. Conf. Abstract and Applied Analysis, eds. Choung, Nirenberg, Tuschke, World Scientific Publishing 2004, p. 139-162.

[4] H. Harbrecht, W. Dahmen and R. Schneider, Adaptive Wavelet Expansion for Integral Equations, in preparation

[5] R. Stevenson, On the compressibility of operators in wavelet coordinates, SIAM J. Math. Anal. 35(5),p. 1110-1132 (2004)

\section{Numerical Solution of Operator Equations with Stochastic Data Christoph Schwab \\ (joint work with Tobias von Petersdorff)}

Strongly elliptic integral equations with stochastic stochastic data are solved numerically. Both, stochastic (Monte-Carlo) and deterministic (sparse tensor product) methods for the approximation of the $k$-th moment of the random solution are analyzed and implemented. Deterministic equations for the $k$-th moment of the random solution are derived and their strong ellipticity and regularity in scales of anisotropic Sobolev spaces are established. Solution algorithms of log-linear complexity (in the number $N$ of degrees of freedom for the mean field problem) based on wavelet compression of the Galerkin stiffness matrix for the mean field 
problem and sparse tensor products of multilevel finite element spaces are analyzed and implemented. Application to the efficient calculation of variances of random solutions to the stochastic Dirichlet and Neumann problems of potential theory via first kind boundary integral equations with stochastic data are presented.

The work generalizes [ST1] and [ST2] for local operator equations.

\section{REFERENCES}

[1] C. Schwab and R.A. Todor: Sparse Finite Elements for elliptic problems with stochastic data Numerische Mathematik 95(2003) 707-734.

[2] Sparse finite elements for stochastic elliptic problems-higher order moments Computing $\mathbf{7 1}$ (2003) 43-63.

\section{Tearing and Interconnecting Domain Decomposition Methods Olaf Steinbach

\author{
(joint work with Ulrich Langer, Günther Of and Walter Zulehner)
}

Domain decomposition methods are a well established tool for the coupling of different partial differential equations, discretization methods such as finite and boundary element methods, and of different (non-matching) finite-dimensional trial spaces and their underlying meshes [4]. In particular Dirichlet domain decomposition methods are based on the coupling of local Dirichlet boundary value problems where the unknown Dirichlet data on the skeleton of the domain decomposition are to be find. The solution of the local Dirichlet boundary value problems define local Dirichlet to Neumann maps including the Steklov-Poincaré operator which can be either expressed by boundary integral operators or by using a domain variational formulation. In both cases we end up with a global Steklov-Poincaré operator equation on the skeleton. The approximation of the local Dirichlet to Neumann maps by using either finite or boundary element methods then leads to a positive definite and symmetric linear system, where the global stiffness matrix is assembled by the local contributions. In this talk we focus on efficiently preconditioned and parallel solution methods to solve the global linear system with almost optimal order in the complexity.

The Finite Element Tearing and Interconnecting (FETI) methods were introduced in [1] as an efficient preconditioned iterative solver for finite element Dirichlet domain decomposition methods. The continuity of the primal variables across the coupling boundaries is formulated as a constraint. Hence, using Lagrange multipliers this leads to a saddle point formulation where the discrete Steklov-Poincaré operators are localized and therefore can be inverted in parallel. Note that for floating subdomains, in particular for subdomains without Dirichlet boundary, due to the non-trivial kernel a suitable pseudo-inverse has to be introduced. After eliminating the primal variables, i.e. the local Dirichlet data, one has to solve the resulting global Schur complement system for the Lagrange multipliers. The corresponding stiffness matrix is symmetric and positive definite and hence the linear system can be solved by using a (projected) conjugate gradient method. 
An appropriate global preconditioner can be build via scaled local Schur complements yielding almost optimal estimates for the spectral condition number of the preconditioned system. Note that this bound is independent of coefficient jumps.

In [2] Boundary Element Tearing and Interconnecting (BETI) methods were introduced as boundary element counter part of the FETI methods. Due to a unified approach of both methods coupled BETI/FETI methods are straightforward [3]. While the original FETI preconditioner is based on the discrete Steklov-Poincaré operator, which is realized by solving local Neumann boundary value problems, the BETI preconditioning methods are based on the use of the hypersingular boundary integral operator which is spectrally equivalent to the discrete finite element/boundary element Steklov-Poincaré operator, and which is already available in the boundary element discretization. Hence we end up with an almost optimal BETI preconditioner to be used in both finite and boundary element tearing and interconnecting domain decomposition methods.

The local Galerkin discretization of boundary integral operators leads to dense stiffness matrices where both the storage and the matrix vector multiplications are quadratic in the number of degrees of freedom belonging to the local subdomain boundaries. Hence one has to use fast boundary element methods (see for example [5]) to obtain an almost linear, up to polylogarithmic terms, complexity. One possible choice is the use of the Fast Multipole Method, other methods are panel clustering, algebraic approximation methods such as Adaptive Cross Approximation or hierarchical matrices, or using wavelets.

Instead of solving the Schur complement system for the Lagrange multipliers, i.e. inverting the discrete Steklov-Poincaré operators locally in each global iteration step, one may solve the saddle point problem by using an appropriate iterative method. Since the discrete Steklov-Poincaré operators are defined via the solution of local Dirichlet boundary value problems, an additional decoupling gives a two-fold saddle point problem to be solved when using boundary elements locally. Note that for floating subdomains the required solvability conditions may be formulated as constraints leading then to a three-fold saddle point problem. However, using a projected iterative method this again is equivalent to a two-fold saddle point problem.

The challenge is now to solve the resulting two-fold saddle point problems by using some preconditioned iterative solution strategies. Following [8] one can apply suitable transformations leading to a positive definite and symmetric linear system when using appropriate inner products. Such an approach requires the use of the previously defined BETI preconditioner as well as the use of local preconditioners for the discrete Steklov-Poincaré operators and the discrete single layer potentials which are involved in the solution of the local Dirichlet problems. As local preconditioners one may use the concept of boundary integral operators of the opposite order [7] or some artificial multilevel methods [6] which are based on the hierarchy already generated by the fast boundary element method. Using those preconditioners one can prove almost optimal spectral equivalence inequalities which are sharp. Therefore the resulting algorithm, i.e. the iterative solution 
of the two-fold saddle point problem using fast boundary element methods locally is almost optimal with respect to the amount of work.

First numerical examples for three-dimensional model problems confirm the theoretical results. A sensitive problem is hereby the choice of optimal parameters needed in the transformation applied to the saddle point problem. In ongoing research we want to apply these methods for more complicated boundary value problems, e.g. in linear elasticity with composed materials, and for complex geometrical data. The coupling of boundary and finite element tearing and interconnecting methods enables us to solve such problems in an efficient way in parallel.

\section{REFERENCES}

[1] C. Farhat, F.-X. Roux: A method of finite element tearing and interconnecting and its parallel solutin algorithm. Int. J. Numer. Meth. Engrg. 32 (1991) 1205-1227.

[2] U. Langer, O. Steinbach: Boundary element tearing and interconnecting methods. Computing 71 (2003) 205-228.

[3] U. Langer, O. Steinbach: Coupled boundary and finite element tearing and interconnecting methods. In: Domain Decomposition Methods in Science and Engineering (R. Kornhuber, R. Hoppe, J. Periaux, O. Pironneau, O. Widlund, J. Xu eds.), Lecture Notes in Computational Science and Engineering, vol. 40, Springer, Heidelberg, pp. 83-97, 2004.

[4] O. Steinbach: Stability estimates for hybrid coupled domain decomposition methods. Lecture Notes in Mathematics 1809, Springer, Heidelberg, 2003.

[5] O. Steinbach: Numerische Näherungsverfahren für elliptische Randwertprobleme. Finite Elemente und Randelemente. B. G. Teubner, Stuttgart, Leipzig, Wiesbaden, 2003.

[6] O. Steinbach: Artificial multilevel boundary element preconditioners. Proc. Appl. Math. Mech. 3 (2003) 539-542.

[7] O. Steinbach, W. L. Wendland: The construction of some efficient preconditioners in the boundary element method. Adv. Comput. Math. 9 (1998) 191-216.

[8] W. Zulehner: Analysis of iterative methods for saddle point problems: a unified approach. Math. Comp. 71 (2002) 479-505.

\section{Some Schwarz Methods for Integral Equations on Surfaces - $h$ and $p$ Versions \\ ERNST P. STEPHAN \\ (joint work with Florian Leydecker and Matthias Maischak)}

Multiplicative and additive Schwarz Methods are presented for the $p$ version of the boundary element method (BEM) applied to first kind integral equations on surfaces. The integral equations under consideration are the weakly singular equation with the single layer potential

$$
V \psi(x):=-\frac{1}{2 \pi} \int_{\Gamma} \frac{\psi(y)}{|x-y|} d s_{y}=f(x), \quad x \in \Gamma=(-1,1)^{2}
$$

and the hypersingular integral equation

$$
W v(x):=\frac{1}{2 \pi} \frac{\partial}{\partial n_{x}} \int_{\Gamma} \frac{\partial}{\partial n_{y}} \frac{v(y)}{|x-y|} d s_{y}=g(x), \quad x \in \Gamma=(-1,1)^{2} .
$$


For (1) the $p$ version of the Galerkin method is performed by use of affine images of Legendre polynomials as boundary elements on a uniform mesh. The resulting stiffness matrix has condition number $\mathcal{O}\left(p^{3}\right)$ where $p$ denotes the maximal polynomial degree of the trial functions on the elements of the mesh. We decompose the trial space into a "coarse space" - spanned by those functions which are piecewise constant in at least one variable - and into subspaces given by polynomials of higher degrees on each element. It is shown that the multiplicative Schwarz method for this stable subspace decomposition has a contraction rate which grows only polylogarithmically with $p$, i.e. the number of Schwarz iterations is only moderately increasing. The proof in [2] is based on a general result for the error propagation operator of the multiplicative Schwarz method given in [3] which uses bounds for the minimal and maximal eigenvalues of the corresponding additive Schwarz operator together with the strengthened Cauchy-Schwarz inequality which bounds the cosin of the angle between the subspaces on the patches.

For (2) the $p$ version of the Galerkin method is done on triangular mesh using special low-energy basis functions together with suitable polynomial extensions of vertex functions and edge functions into triangles. We present from [1] an iterative substructuring method which is based on splitting of the trial space into wire-basket functions and interior functions (bubbles). The resulting additive Schwarz preconditioner has block-diagonal structure and the condition number of the Schwarz operator behaves like $\mathcal{O}\left(\log p^{3}\right)$.

Finally, the $h$ version for (2) with piecewise linear, continuous trial functions is considered on a sequence of uniform, triangular meshes. Here, the multigrid algorithm (V cycle with one Jacobi smoother per level) has bounded condition number. On the other hand the condition number of the multilevel additive Schwarz operator grows logarithmically in $h^{-1}$ with the mesh size $h$ of the finest level.

\section{REFERENCES}

[1] N. Heuer and F. Leydecker and E.P. Stephan. Iterative substructuring method for the $p$ version of the BEM on triangular meshes. Report \#60 (2003) Inst. Angew. Mathematik, Universität Hannover.

[2] M. Maischak. FEM/BEM methods for Signorini-type problems, error analysis, adaptivity, preconditioners. Habilitation thesis, 2001 Inst. Angew. Mathematik, Universität Hannover.

[3] M. Maischak and E.P. Stephan and T. Tran. Multiplicative Schwarz algorithms for the Galerkin boundary element method. SIAM J. Numer. Anal., 38, 1243-1268, 2000.

\section{A spectral method for integral formulations of potential and high-frequency scattering problems}

\section{Johannes TAUSCH}

A variety of methods exist to handle dense matrix problems that arise in the boundary element method. For Green's functions with $1 / r$ behavior, which are typical in potential or elasticity theory, the Fast Multipole Method, Panel clustering and Wavelets have been shown to be asymptotically optimal. That is, the complexity of a matrix-vector multiplication is order $N$ while the convergence rate 
of the discretization scheme is preserved, see, e.g. [3, 2, 4]. For scattering problems, the size of the scatterer, measured in wavelengths, is an additional factor that influences the computational cost and accuracy.

This talk discusses a different approach, which is based on fast Fourier transforms and is thus more close to the previous work of Phillips and White [1]. However, in this work we will approximate the Green's function by a truncated Fourier series, which considerably simplifies the treatment of the nearfield. Furthermore, the convergence of the method can be improved with a decomposition of the Greens function into a local and a smooth part.

Fast computation of the potential. Consider the layer potential

$$
(K g)(x):=\int_{S} G(x-y) g(y) d S_{y}, \quad x \in S
$$

where the Green's function $G$ depends only on the difference of the field point $x$ and the source point $y$. We assume the problem is scaled that the surface $S$ is contained in the unit cube $[0,1]^{3}$. In the case that the Green's function is well approximated by the truncated Fourier series $G_{N}$

$$
G_{N}(r):=\sum_{|k| \leq N} \widehat{G}_{k} \exp (\pi i k \cdot r)
$$

the approximate potential is

$$
\phi_{N}(x)=\int_{S} G_{N}(x-y) g(y) d S_{y}=\sum_{|k|<N} \exp \left(\pi i k^{T} x\right) \widehat{d}_{k}
$$

where $\widehat{d}_{k}=\widehat{G}_{k} \widehat{g}_{k}$ and

$$
\widehat{g}_{k}=\int_{S} \exp \left(-\pi i k^{T} y\right) g(y) d S_{y} .
$$

Thus the potential evaluation using the Fourier series consists of three stages

(1) Compute the Fourier coefficients $\widehat{g}_{k}$ in (3).

(2) Multiply $\widehat{d}_{k}:=\widehat{G}_{k} \widehat{g}_{k}$.

(3) Evaluate the Fourier series (2).

Stage 2 obviously involves $O\left(N^{3}\right)$ operations, the other two stages can be performed rapidly using Fast Fourier Transforms. For brevity we discuss Stage 1 only as Stage 3 can be treated in an analogous manner.

To compute the $\widehat{g}_{k}$ 's efficiently, divide the unit cube in an axiparallel mesh of sidelength $1 / N$ and let $S_{l}$ be the piece of the surface that intersects with the $l$ th subcube. Then the Fourier coefficients of $g$ can be written as

$$
\widehat{g}_{k}=\sum_{l} \exp \left(\frac{-\pi i k^{T} l}{N}\right) \int_{S_{l}} \exp \left(-\pi i k^{T}\left(y-x_{l}\right)\right) g(y) d S_{y},
$$

where $x_{l}$ is the center of the $l$ th subcube. To approximate the integrand, replace in each term the exponential function by a truncated Taylor series of order $p$, this 
gives

$$
\widehat{g}_{k} \approx \sum_{|\alpha| \leq p}(-\pi i k)^{\alpha} \sum_{l} \exp \left(\frac{-\pi i k^{T} l}{N}\right) \int_{S_{l}} \frac{\left(y-x_{l}\right)^{\alpha}}{\alpha !} g(y) d S_{y},
$$

where $\alpha \in \mathbf{N}^{3}$ is a multi-index. The last integrals are the moments of $g$. In matrix form, the (approximate) coefficient vector $\widehat{g}$ is given by

$$
\widehat{g}=\sum_{|\alpha| \leq p} K_{\alpha} F m_{\alpha}
$$

where $m_{\alpha}$ is the vector of moments of $g$ and $K_{\alpha}$ is a diagonal matrix with the factors $(-\pi i k)^{\alpha}$. Thus the computation of $\widehat{g}$ involves $O\left(p^{3}\right)$ FFTs. Since $C_{l}$ is smaller than a wavelength of the highest Fourier mode, it suffices to use a small value of $p$.

Improving the convergence of the Fourier Series. When the kernel in (1) is the Green's function of an elliptic PDE then the Fourier series approximation converges slowly because of the singularity at $r=0$. In this case convergence can be improved by decomposing the Green's function into a local and a smooth part. To that end, consider the Green's function associated with the Laplacian, whose Fourier transform can be written as

$$
\frac{1}{|\xi|^{2}}=\int_{0}^{\infty} \exp \left(-|\xi|^{2} t\right) d t=\int_{0}^{\delta} \exp \left(-|\xi|^{2} t\right) d t+\int_{\delta}^{\infty} \exp \left(-|\xi|^{2} t\right) d t
$$

for some $\delta>0$. The first term is the local, the second term is the smooth part. Both parts have closed forms in physical space, for instance, the smooth part is

$$
G_{\delta}^{S}(r)=\frac{1}{4 \pi|r|} \operatorname{erf}\left(\frac{|r|}{2 \sqrt{\delta}}\right)
$$

For this kernel the FFT-based method can be employed. The remaining local part can be evaluated by Taylor series expansions. As it can be shown that

$$
\left(K_{\delta}^{L} g\right)(x):=\int_{S} G_{\delta}^{L}(x-y) g(y) d S_{y}=\sqrt{\delta} g(x)+O\left(\delta^{3 / 2}\right) .
$$

the approximate evaluation of this potential reduces to a diagonal operation.

The talk will discuss choices of the parameters $\delta$ and $N$ that will lead to asymptotically optimal schemes and present numerical results obtained with BEM formulations of the Laplace and high frequency Helmholtz equation.

\section{REFERENCES}

[1] J. Phillips and J. White. A precorrected-FFT method for electrostatic analysis of complicated 3-d structures. IEEE Trans. Circuits and Systems, 16(10):1059-1073, 1997.

[2] S.A. Sauter. Variable order panel clustering. Computing, 64(3):223-277, May 2000.

[3] Reinold Schneider. Multiscalen- und Wavelet- Matrixkompression: Analysisbasierte Methoden zur effizienten Loesung grosser vollbesetzter Gleichungssysteme. Teubner, Stuttgart, 1998.

[4] Johannes Tausch. The variable order fast multipole method. Computing, 2004. To appear. 


\section{Matrix approximations and solvers using tensor products and non-standard wavelet transforms related to irregular grids \\ EugENE TYRTYSHNIKOV \\ (joint work with Ivan Oseledets)}

Let $R(X)$ be considered as an approximation of $X$. Assume that $R(X)$ is a nonlinear Lipschitz operator on $n \times n$ matrices such that

$$
\|(X-R(X))-(Y-R(Y))\| \leq M\|X-Y\| .
$$

On iteration $k$, the Newton method transforms $X_{k-1}=R\left(Z_{k-1}\right)$ to a new guess $X_{k}=R\left(Z_{k}\right)$ (for some $Z_{k-1}$ and $Z_{k}$ ):

$$
Z_{k}=2 X_{k-1}-X_{k-1} A X_{k-1}, \quad X_{k}=R\left(Z_{k}\right)
$$

Theorem. Assume that $R\left(A^{-1}\right)=A^{-1}$. Then for any initial guess $X_{0}=$ $R\left(X_{0}\right)$ sufficiently close to $A^{-1}$, the matrices $X_{k}$ in $(*)$ converge to $A^{-1}$ quadratically:

$$
\left\|A^{-1}-X_{k}\right\| \leq(1+M)\|A\|\left\|A^{-1}-X_{k-1}\right\|^{2}, \quad k=1,2, \ldots .
$$

Consider an important example of $R(x)$. Let $\|\cdot\|$ be any unitary invariant norm (spectral, Frobenius, etc.) and $\Pi_{r}(A)$ the best $r$-rank approximation:

$$
\rho_{r+1}(A) \equiv\left\|A-\Pi_{r}(A)\right\|=\min _{\operatorname{rank} B \leq r}\|A-B\| .
$$

Let $L$ be a linear invertible operator on $n \times n$ matrices. Fix some $r$ and define

$$
R(A)=L^{-1}\left(\Pi_{r}(L(A))\right) .
$$

The Lipschitz property of $R$ is proved as follows:

$$
\|R(X)-R(Y)\| \leq\left\|L^{-1}\right\|\left\|\Pi_{r}(L X)-\Pi_{r}(L Y)\right\| \leq\left\|L^{-1}\right\|\|L\|\|X-Y\| .
$$

Low-tensor-rank approximations fit this framework:

$$
A=\left[a_{i j}^{k m}\right], \quad 1 \leq i, j, k, m \leq p . \quad \Rightarrow \quad L(A)=\left[b_{i j}^{k m}\right], \quad b_{i j}^{k m}=a_{i k}^{j m} .
$$

Tensor rank of $A$ coincides with rank of $L(A)$ (Van Loan - Pitsianis).

Remarkable generalizations of Toeplitz (Hankel, Cauchy, etc.) matrices are all related with low rank of $L(A)=A U-V A$ for special matrices $U, V$. Our theorem generalizes a result obtained for this particular case of $L(A)$ by V. Y. Pani and Y. Rami.

Dense large-scale matrices coming from integral equations and tensor-product grids can be approximated by a sum of Kronecker products with further sparsification of the factors via discrete wavelet transforms, which results in reduced storage and computational costs $[3,5,6]$ and also in good preconditioners in the case of uniform one-dimensional grids [1]. However, irregular grids lead to a loss of approximation quality and, more significantly, to a severe deterioration in efficiency of the preconditioners that have been considered previously (using a sparsification of the inverse to one Kronecker product or an incomplete factorization approach). We propose to use non-standard wavelet transforms related to the irregular grids 
involved and we show, using numerical examples, that the new transforms provide better compression than the Daubechies wavelets [2]. A further innovation is a scaled two-level circulant preconditioner that performs well on irregular grids [2]. Also, we have encouraging results concerning a truncation algorithm for approximation of the inverse matrices of low Kronecker rank [4]. Our proposed approximation and preconditioning techniques have been applied to a hypersingular integral equation modelling flow around a thin aerofoil, and make it possible to solve linear systems with more than 1 million unknowns in 5-10 minutes even on a personal computer $[2,4]$.

\section{REFERENCES}

[1] J. M. Ford, E. E. Tyrtyshnikov, Combining Kronecker product approximation with discrete wavelet transforms to solve dense, function-related systems, SIAM J. Sci. Comp., Vol. 25, No. 3 (2003), 961-981.

[2] J. M. Ford, I. V. Oseledets, E. E. Tyrtyshnikov, Matrix approximations and solvers using tensor products and non-standard wavelet transforms related to irregular grids, Rus. J. Numer. Anal. and Math. Modelling, Vol. 19, No. 2 (2004), 185-204.

[3] W. Hackbusch W., B. N. Khoromskij, E. E. Tyrtyshnikov, Hierarchical Kronecker tensorproduct approximations, Max-Planck-Institut für Mathematik in den Naturwissenschaften, Leipzig, Preprint No. 35, 2003.

[4] I. V.Oseledets, E. E. Tyrtyshnikov, Approximate inversion of matrices with solution of the hypersingular integral equation, J. Comput. Math. and Math. Physics, to appear.

[5] E. E. Tyrtyshnikov, Tensor approximations of matrices generated by asymptotically smooth functions, Sbornik: Mathematics, Vol. 194, No. 6 (2003), pp. 147-160.

[6] E. E. Tyrtyshnikov, Kronecker-product approximations for some function-related matrices, Linear Algebra Appl., 379 (2004), 423-437.

\section{On J. Radon's convergence proof of Neumann's method with double layer potentials}

\section{WOLFGANG L. WENDLAND}

This lecture with the same title was also given at the Geomathematics Oberwolfach Conference.

C.F. Gauss proposed for the construction of the solution $u$ to the Dirichlet problem of the Laplacian with given boundary values $\varphi$ the use of a double layer potential

$$
u(x)=-\frac{1}{4 \pi} \int_{\Gamma} \mu(y) d \Omega_{x}(y) \quad \text { for } \quad x \in \Omega
$$

which leads with the jump relation to C. Neumann's boundary integral equation

$$
\mu=L \mu+\varphi
$$

for the boundary double layer charge $\mu$, where

$$
(L \mu)(x)=-\frac{1}{4 \pi} \int_{\Gamma}(\mu(y)-\mu(x)) d \Omega_{x}(y) \quad \text { for } \quad x \in \Gamma
$$


is the regularized double layer potential operator. Here $\Omega_{x}(E)$ is the solid angle from $x$ for measurable $E \subseteq \Gamma$, a signed Radon measure and an absolutely continuous set function introduced by J. Radon in $[18,19]$. For piecewise smooth $\Gamma$ including corners and edges, here a review is given on Radon's treatment of the boundary integral equation and on the extensions by V. Maz'ya, J. Kral, D. Medkova and O. Jansen if the equation is considered on the Banach space of continuous functions $\mu$ on $\Gamma$. For the corresponding two-dimensional problem, J. Radon in his famous papers defined closed boundary curves of bounded rotation and showed that for such curves without sharp cusps, the essential norm of $L$ generated by the supremum norm is less than 1 , he also showed the relation between eigenvalues of $L$ and exterior and interior Dirichlet integrals of the eigensolution potentials, which implies that the spectral radius of $L$ is less than 1 . Hence, Neumann's classical successive approximation can be applied for solving the boundary integral equation iteratively. In three dimensions, however, the corresponding results are by no means complete yet. Here J. Kral and D. Medkova have introduced the family of weighted supremum norms

$$
\|\mu\|_{C_{w}^{0}(\Gamma)}:=\sup _{x \in \Gamma}|w(x) \mu(x)|
$$

with a strictly positive, bounded weight function $w(x)$ in order to generalize results by V. Maz'ya [11], J. Kral [9] and the author [23] for $\Gamma \in \mathbb{R}^{3}$. As it turns out, it can be proved that the essential spectral radius satisfies $r_{\text {ess }}(L)<1$ for piecewise smooth $\Gamma$ in the following cases:

With $w \equiv 1$ for convex $\Gamma[15,16]$, for $C^{1+\alpha}$-smooth (also nonconvex) $\Gamma$ [17], if $\Gamma$ has edges with nonvanishing opening angle but no corners [4]; if $\Gamma$ has corners and edges such that $\lim _{\delta \rightarrow 0+}\left\{\sup _{x \in \Gamma} \frac{1}{4 \pi} \int_{0<|x-y| \leq \delta}\left|d \Omega_{x}(y)\right|\right\}<\frac{1}{2}$ holds $[2,3,8,9,23]$ and if $\Gamma$ has isolated conical points [6]. If $\Gamma$ is a so-called rectangular surface then a sectorially constant weight function $w$ is constructed in $[1,10]$. For general polyhedral boundary, however, the existence of $w$ was shown in $[20,21]$ but the proof is not constructive. On the other hand, O. Hansen constructs a sectorially constant function $w$ near a polyhedral corner under additional conditions on the edge angles [7]. With his construction, a rather large class of polyhedrons become admissible whereas for a general polyhedron, the construction of a piecewise constant weight function is still not known. D. Medkova showed in $[12,13]$ the invariance of $r_{\text {ess }}(L)$ under $\mathbb{R}^{3}$ diffeomorphisms which are conformal at the corner point.

In all these cases the Fredholm alternative is valid for the boundary integral equation of the second kind, also for piecewise constant trial functions on a triangulation of $\Gamma$ which is compatible with the weight function $w$. Moreover, stability and convergence of the classical collocation (or panel) method can be proved [10].

If boundary element Galerkin methods in the $L^{2}(\Gamma)$ setting are used, then only for convex polyhedrons and for polyhedrons satisfying specific edge conditions, the spectral radius generated by the $L^{2}$ norm is known to be less than $1[5,14]$, whereas for general polyhedrons the corresponding result again is yet not known. 
If, however, the boundary integral equation is considered on the trace space $H^{\frac{1}{2}}(\Gamma)$ and is treated with an appropriate Galerkin-Petrov method, then an appropriate norm of $L$ on $H^{\frac{1}{2}}(\Gamma)$ is less than 1 and Neumann's classical successive approximation converges for the corresponding Petrov-Galerkin equations. Moreover, the method is stable and convergent [22]. These properties are of great value for practical computations and some corresponding results from industrial applications are presented.

\section{REFERENCES}

[1] T.S. Angell, R.E. Kleinman and J. Kral: Layer potentials on boundaries with corners and edges. Cas. Pěst. Mat. 113 (1988) 387-402.

[2] Yu D. Burago and V.G. Maz'ya: Potential theory and function theory for irregular regions. Zap. Naučn. Sem. LOMI 3 (1967) 1-152 (in Russian); Seminars in Mathematics V.A. Steklov Math. Inst. Leningrad (1969) 1-86

[3] Yu D. Burago, V.G. Maz'ya and V.D. Sapozhnikova: On the double layer potential for nonregular domains. Dokl. Akad. Nauk. SSSR 147 (1962) 423-525; Sov. Math. Dokl. 3 (1962) 1640-1642.

[4] T. Carleman: Über das Neumann-Poincarésche Problem für ein Gebiet mit Ecken. Inaugural-Dissertation. Uppsala 1916.

[5] E. Fabes, M. Sand, J.K. Seo: The spectral radius of the classical layer potentials on convex domains. In: Proc. IMA Conference Chicago 1990; Springer-Verlag, New York. IMA Vol. Math. Appl. 142 (1992) 129-137.

[6] N.V. Grachev and V.G. Maz'ya: On the Fredholm radius for operators of the double layer potential type on piecewise smooth boundaries. Vestn. Leningrad. Univ. 19 (1986) 60-64.

[7] O. Hansen: On the essential norm of the double layer potential on polyhedral domains and the stability of the collocation method. J. Integral Equations Appl. 13 (2001) 207-235.

[8] J. Kral: The Fredholm method in potential theory. Trans. Amer. Math. Soc. 125 (1966) 511-547.

[9] J. Kral: Integral Operators in Potential Theory. Lecture Notes in Mathematics 823 SpringerVerlag, Berlin 1980.

[10] J. Kral and W.L. Wendland: On the applicability of the Fredholm-Radon method in potential theory and the panel method. In: Panel Methods in Fluid Mechanics with Emphasis in Aerodynamics (J. Ballmann et al. eds.) Notes on Numerical Fluid Mechanics Vieweg-Verlag 21 (1988) 120-136.

[11] V.G. Maz'ya: Boundary integral equations. In: Encyclopaedia of Mathematical Sciences 27, Analysis IV (V.G. Maz'ya, S.M. Nikoloski eds.) Springer-Verlag Berlin (1991) 127-222.

[12] D. Medková: Invariance of the Fredholm radius of the Neumann operator. Mathematica Bohemica 115 (1990) 147-164.

[13] D. Medková: On essential norm of the Neumann operator. Mathematica Bohemica 117 (1992) 393-408.

[14] I. Mitrea: Spectral radius properties for layer potentials associated with the elastostatics and hydrostatics equations in nonsmooth domains. J. Fourier Anal. Appl. 5 (1999) 385-408.

[15] C. Neumann: Zur Theorie des logarithmischen und des Newtonschen Potentials. Ber. Verh. Math.-Phys. Classe Königl. Sächs. Akad. Wiss. Leipzig 23 (1870) 49-56; 264-321.

[16] C. Neumann: Über die Methode des Arithmetischen Mittels, Hirzel, Leipzig 1887 (erste Abh.), 1888 (zweite Abh.).

[17] J. Plemelj: Potentialtheoretische Untersuchungen. Teubner-Verlag, Leipzig 1911.

[18] J. Radon: Über lineare Funktionaltransformationen und Funktionalgleichungen. Sitzber. Akad. Wiss. Wien 128 (1919) 1083-1121.

[19] J. Radon: Über die Randwertaufgaben beim logarithmischen Potential. Sitzber. Akad Wiss. Wien 128 (1919) 1123-1167. 
[20] A. Rathsfeld: The invertibility of the double layer potential in the space of continuous functions defined on a polyhedron. The panel method. Applicable Analysis 45 (1992) 1-4, $135-177$.

[21] A. Rathsfeld: The invertibility of the double layer potential in the space of continuous functions defined on a polyhedron. The panel method. Erratum. Applicable Analysis 56 (1995) 109-115.

[22] O. Steinbach and W.L. Wendland: On C. Neumann's method for second order elliptic systems in domains with non-smooth boundaries. J. Math. Anal. Appl. 262 (2001) 733-748.

[23] W.L. Wendland: Zur Behandlung von Randwertaufgaben im $\mathbb{R}_{3}$ mit Hilfe von Einfach- und Doppelschichtpotentialen. Numer. Math. 11 (1968) 380-404. 


\section{Participants}

\author{
Dr. Mario Bebendorf \\ bebendorf@math .uni-leipzig.de \\ Fakultät für Mathematik/Informatik \\ Universität Leipzig \\ Augustusplatz 10/11 \\ 04109 Leipzig
}

Prof. Dr. Carsten Carstensen

cc@math.hu-berlin.de

Institut für Mathematik

Humboldt-Universität zu Berlin

Unter den Linden 6

10099 Berlin

Prof. Dr. Simon N. ChandlerWilde

S.N.Chandler-Wilde@reading.ac.uk

Department of Mathematics

University of Reading

WhiteKnights

P.O.Box 220

GB-Reading, RG6 6AX

Prof. Dr. Ivan G. Graham

i.g.graham@bath.ac.uk

igg@maths. bath.ac.uk

Department of Mathematical Sciences

University of Bath

Claverton Down

GB-Bath, BA2 7AY

\section{Dr. Lars Grasedyck}

lgr@mis.mpg.de

Max-Planck-Institut für Mathematik

in den Naturwissenschaften

Inselstr. 22 - 26

04103 Leipzig
Prof. Dr. Wolfgang Hackbusch

wh@mis.mpg.de

Max-Planck-Institut für Mathematik in den Naturwissenschaften

Inselstr. 22 - 26

04103 Leipzig

Prof. Dr. Ralf Hiptmair

hiptmair@sam.math.ethz.ch

Seminar für Angewandte Mathematik

ETH-Zentrum

Rämistr. 101

CH-8092 Zürich

\section{Dr. Boris N. Khoromskij}

bokh@mis.mpg.de

Boris. Khoromskij@mis.mpg. de

Max-Planck-Institut für Mathematik

in den Naturwissenschaften

Inselstr. 22 - 26

04103 Leipzig

\section{Prof. Dr. Rainer Kreß}

kress@math . uni-goettingen.de

Institut für Numerische

und Angewandte Mathematik

Universität Göttingen

Lotzestr. 16-18

37083 Göttingen

\section{Dr. Christian Lage}

cl@numiracle.com

Beckerstr. 11

12157 Berlin

Prof. Dr. Ulrich Langer

ulanger@numa . uni-linz . ac . at

Institut für Numerische Mathematik

Johannes Kepler Universität Linz

Altenbergstr. 69

A-4040 Linz 
Prof. Dr. Giovanni Monegato

giovanni.monegato@polito.it

Dipartimento di Matematica

Politecnico di Torino

Corso Duca degli Abruzzi, 24

I-10129 Torino

Prof. Dr. Sergej Rjasanow

rjasanow@num.uni-sb.de

FR 6.1 - Mathematik

Universität des Saarlandes

Postfach 151150

66041 Saarbrücken

Prof. Dr. Stefan A. Sauter

stas@amath.unizh.ch

Institut für Mathematik

Universität Zürich

Winterthurerstr. 190

CH-8057 Zürich

Prof. Dr. Reinhold Schneider

rs@numerik.uni-kiel.de

Lehrstuhl für Scientific Computing

Universitäts-Hochhaus 9 Stock

Christian-Albrechts-Platz 4

24098 Kiel

Prof. Dr. Christoph Schwab

schwab@sam.math.ethz.ch

schwab@math.ethz.ch

Seminar für Angewandte Mathematik

ETH-Zentrum

Rämistr. 101

CH-8092 Zürich

\section{Dr. Olaf Steinbach}

steinbach@mathematik. uni-stuttgart.de

Institut für Angewandte Analysis

und Numerische Simulation

Universität Stuttgart

Pfaffenwaldring 57

70569 Stuttgart

Prof. Dr. Ernst Peter Stephan

stephan@ifam.uni-hannover.de

Institut für Angewandte Mathematik

Universität Hannover

Welfengarten 1

30167 Hannover

Prof. Dr. Johannes Tausch

tausch@mail.smu.edu

Dept. of Mathematics

Southern Methodist University

Clements Hall

Dallas, TX 75275-0156

USA

Prof. Dr. Eugene Tyrtyshnikov

tee@inm.ras.ru

Institute of Numerical Mathematics

of the Russian Academy of Sciences

Ul. Gubkina 8

Moscow 119991

RUSSIA

Prof. Dr.-Ing. Wolfgang L. Wendland

wendland@mathematik. uni-stuttgart. de

Institut für Angewandte Analysis

und Numerische Simulation

Universität Stuttgart

Pfaffenwaldring 57

70569 Stuttgart 